Rodrigo Pinto Pedrosa

\title{
Prevalência e importância cardiovascular dos distúrbios respiratórios do sono na miocardiopatia hipertrófica
}

Tese apresentada à Faculdade de Medicina da Universidade de São Paulo para obtenção do título de Doutor em Ciências

Área de concentração: Pneumologia Orientador: Prof. Dr. Geraldo Lorenzi-Filho Co-orientador: Prof. Dr. Luciano Ferreira Drager 
Dados Internacionais de Catalogação na Publicação (CIP)

Preparada pela Biblioteca da

Faculdade de Medicina da Universidade de São Paulo

reprodução autorizada pelo autor

\section{Pedrosa, Rodrigo Pinto}

Prevalência e importância cardiovascular dos distúrbios respiratórios do sono na miocardiopatia hipertrófica / Rodrigo Pinto Pedrosa. -- São Paulo, 2010.

Tese(doutorado)--Faculdade de Medicina da Universidade de São Paulo.

Programa de Pneumologia.

Orientador: Geraldo Lorenzi-Filho.

Co-orientador: Luciano Ferreira Drager.

Descritores: 1.Apneia do sono tipo obstrutiva 2.Prevalência 3.Cardiomiopatia hipertrófica 4.Fibrilação atrial

USP/FM/DBD-400/10 


\section{Dedicatória}

Aos meus amados pais, Levi e Albania, por tudo que me ensinaram e pelo apoio durante toda minha vida.

À Marcia, meu amor, pelo apoio e companheirismo. 


\section{Agradecimentos}

Ao Prof. Dr. Geraldo Lorenzi-Filho, grande mentor, que me ensinou de forma simples e humilde a ser um pesquisador.

Ao Dr. Luciano Ferreira Drager, responsável por me introduzir na área da medicina do sono e pela grande colaboração na minha formação profissional.

Ao Dr. Pedro Rodrigues Genta, pela paciência nos ensinamentos de estatística e medicina do sono.

A Aline, grande colaboradora nessa pesquisa.

Aos demais colegas do Laboratório do Sono do InCor, pelo apoio durante esses anos. 
Aos colegas Dr. Edmundo Arteaga, Dr. Murillo de Oliveira Antunes e Dr. Afonso Matsumoto, do setor de Cardiomiopatias do InCor, pela imensa colaboração.

Ao Prof. Dr. Mário Terra Filho, coordenador da pós-graduação em Pneumologia, pelo apoio acadêmico durante esses anos.

Ao Prof. Dr. Francisco Vargas, titular da Disciplina de Pneumologia, por me permitir uma pós-graduação de excelência. 


\section{Sumário}

Lista de abreviaturas

Lista de tabelas

Lista de figuras

Resumo

Summary

1

INTRODUÇÃO

1.1 Miocardiopatia hipertrófica......................................

1.2 Distúrbios respiratórios do sono...............................06

1.2.1 Apneia obstrutiva do sono........................................

1.2.2 Apneia central do sono e Respiração de Cheyne-

Stokes.

2

RACIONAL DO ESTUDO.

3

OBJETIVOS.

4

CASUÍSTICA E MÉTODOS. 
4.1 População estudada...........................................18

4.2 Avaliação clínica................................................19

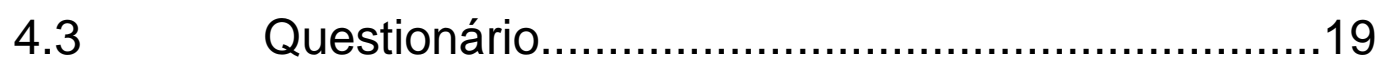

4.4 Bioquímica e eletrocardiograma............................20

4.5 Monitorização cardiorrespiratória noturna.............20

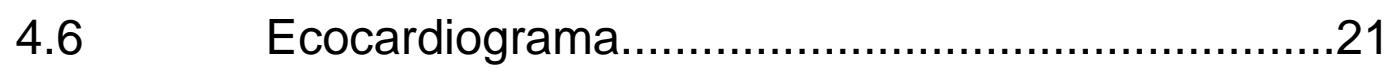

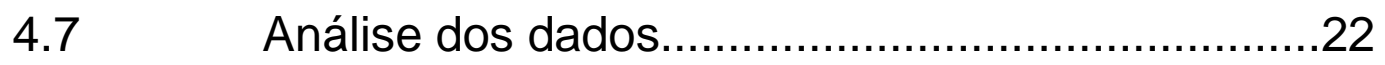

$5 \quad$ RESULTADOS

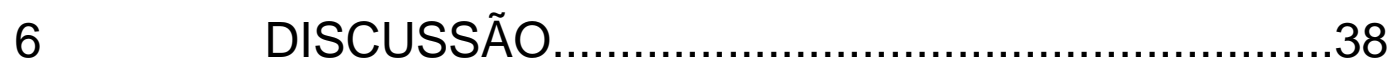

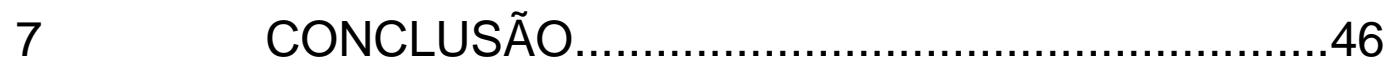

$8 \quad$ ANEXOS

8.1 Anexo A - Termo de consentimento livre e esclarecido........................................................... 49

8.2 Anexo B - Questionário de Epworth.......................53

8.3 Anexo C - Artigo publicado....................................54 


\section{Lista de Abreviaturas}

AOS Apneia obstrutiva do sono

AT1

Angiotensina 1

CF IC Classe funcional da insuficiência cardíaca

CPAP Pressão contínua positiva de vias aéreas superiores

DP Desvio padrão

ECA Enzima conversora de angiotensina

FA Fibrilação atrial

FE Fração de ejeção

IAH Índice de apneia-hipopneia

IC Intervalo de confiança

IMC Índice de massa corpórea

MH Miocardiopatia hipertrófica

NYHA New York Heart Association

PA Pressão arterial

RCS-ACS Respiração de Cheyne-Stokes - Apneia central do sono

$\mathrm{SatO}_{2} \quad$ Saturação da oxi-hemoglobina 
VE Ventrículo esquerdo

VSVE Via de saída do ventrículo esquerdo 


\section{Lista de Tabelas}

Tabela 1

Fatores de risco para morte súbita na miocardiopatia hipertrófica....................................04

Tabela 2

Características clínicas, bioquímicas e medicações da população estudada total e segundo a presença ou ausência de apneia obstrutiva do sono..................................................27

Tabela 3

Características laboratoriais e ecocardiográficas da população estudada total e segundo a presença ou ausência de apneia obstrutiva do sono....................................................................30

Tabela 4

Exame do sono da população estudada total e segundo a presença ou ausência de apneia obstrutiva do sono. 32

Tabela 5

Regressão logística entre a presença de fibrilação atrial com variáveis antropométricas, diagnóstico de hipertensão arterial, variáveis ecocardiográficas e do exame do sono................36

Tabela 6

Regressão logística múltipla entre a presença de fibrilação atrial com as variáveis significativas da análise univariada. 


\section{Lista de Figuras}

Figura 1

Registro polissonográfico de um paciente com apneia obstrutiva do sono. .07

Figura 2

Registro polissonográfico de um paciente com Respiração de Cheyne-Stokes..............................12

Figura 3

Diagrama de fluxo de pacientes. .25

Figura 4

Diâmetro do átrio esquerdo em pacientes com miocardiopatia hipertrófica sem e com apneia

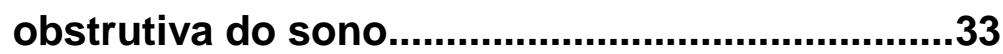

Figura 5

Diâmetro da aorta ascendente em pacientes com miocardiopatia hipertrófica sem e com apneia obstrutiva do sono. .34

Figura 6

Presença de fibrilação atrial em pacientes com miocardiopatia hipertrófica sem e com apneia obstrutiva do sono. .35 


\section{RESUMO}

Pedrosa RP. Prevalência e importância cardiovascular dos distúrbios respiratórios do sono em pacientes com miocardiopatia hipertrófica. Tese: Faculdade de Medicina, Universidade de São Paulo, São Paulo, São Paulo; 2010.

Introdução: A miocardiopatia hipertrófica é a mais frequente doença cardiovascular de origem genética e está associada a arritmias e morte cardiovascular. O aumento do átrio esquerdo e a fibrilação atrial são considerados marcadores de morte por insuficiência cardíaca em pacientes com miocardiopatia hipertrófica. A apneia obstrutiva do sono é o distúrbio respiratório do sono mais comum, caracterizando-se por episódios recorrentes de colapso parcial ou total das vias aéreas superiores durante o sono. A apneia obstrutiva do sono é muito prevalente entre as populações com doença cardiovascular, como hipertensão arterial e insuficiência cardíaca, e está associada a remodelamento cardíaco e arritmias. Objetivos: O objetivo deste estudo foi determinar a prevalência dos distúrbios respiratórios do sono em pacientes com miocardiopatia hipertrófica e avaliar a associação da apneia obstrutiva do sono com o remodelamento cardíaco (ventricular e atrial) e fibrilação atrial em pacientes com miocardiopatia hipertrófica. Métodos: Foram estudados pacientes consecutivos estáveis clinicamente, com um diagnóstico confirmado de miocardiopatia hipertrófica acompanhados no Instituto do Coração do Hospital das Clínicas da Faculdade de Medicina da Universidade de São Paulo. 
Os pacientes foram submetidos à avaliação clínica, questionário de sonolência, bioquímica sanguínea, ecocardiograma e monitorização respiratória noturna com poligrafia portátil. Foi utilizado um valor de corte de 15 e 30 apneias e hipopneias por hora de registro para o diagnóstico de apneia obstrutiva do sono e apneia obstrutiva do sono grave, respectivamente. Resultados: Foram avaliados 80 pacientes consecutivos com miocardiopatia hipertrófica. Apneia obstrutiva do sono foi diagnosticada em 32 pacientes (40\%). Apneia obstrutiva do sono grave esteve presente em 17 pacientes (21\%). Pacientes com apneia obstrutiva do sono foram significativamente mais velhos (56 [41-64] vs. 39 [30-53] anos, $p<$ 0,001), apresentaram maior índice de massa corporal $(28,2 \pm 3,5$ vs. $25,2 \pm 5,2$ $\mathrm{Kg} / \mathrm{m}^{2}, \mathrm{p}<0,01$ ), maior dimensão do átrio esquerdo (45 [42-53] vs. 41 [39-47] $\mathrm{mm}, \mathrm{p}=0.01)$ e maior diâmetro da aorta (34 [30-37] vs. 29 [28-32] mm, $\mathrm{p}<$ 0,001) em comparação com pacientes sem apneia obstrutiva do sono. Dois modelos de regressão linear múltipla para identificar os fatores associados ao aumento do átrio esquerdo e da aorta ascendente mostraram que o índice de apneia e hipopneia foi a única variável associada ao aumento atrial $(p=0,05)$ e da aorta $(p=0,01)$, respectivamente. A fibrilação atrial permanente esteve presente em $31 \%$ vs. $6 \%$ dos pacientes com e sem apneia obstrutiva do sono, respectivamente $(p<0,01)$. A apneia obstrutiva do sono $(p=0,03)$ e o diâmetro do átrio esquerdo $(p=0,03)$ foram os únicos fatores independentemente associados à fibrilação atrial em um modelo multivariado. Conclusão: A apneia obstrutiva do sono é muito prevalente em pacientes com miocardiopatia hipertrófica e está associada com aumento do átrio esquerdo e da aorta 
ascendente. A apneia obstrutiva do sono está independentemente associada à fibrilação atrial, um fator de risco para óbito cardiovascular nesta população.

Descritores: 1. Apneia do sono tipo obstrutiva; 2. Prevalência; 3. Cardiomiopatia hipertrófica; 4. Fibrilação atrial. 


\section{SUMMARY}

Pedrosa RP. Prevalence and cardiovascular importance of sleep disordered breathing in patients with hypertrophic cardiomyopathy. [Thesis].

São Paulo: "Faculdade de Medicina, Universidade de São Paulo"; 2010.

Background: Hypertrophic cardiomyopathy is the most common genetic cardiovascular disease and is associated with arrhythmias and cardiovascular death. Left atrial enlargement and atrial fibrillation are considered markers for death due to heart failure in patients with hypertrophic cardiomyopathy. Obstructive sleep apnea is the most common sleep disordered breathing and is characterized by recurrent episodes of partial or complete collapse of the upper airway during sleep. Obstructive sleep apnea is extremely prevalent among populations with cardiovascular disease, such as systemic hypertension and heart failure and is independently associated with heart remodelling and arrhythmias. Objectives: The aim of this study was to determine the prevalence of sleep disordered breathing in consecutive patients with hypertrophic cardiomyopathy and evaluate the association of obstructive sleep apnea with heart remodelling (ventricular and atrial) and with atrial fibrillation in patients with hypertrophic cardiomyopathy. Methods: We studied consecutive clinically stable patients with a confirmed diagnosis of hypertrophic cardiomyopathy followed in the Heart Institute - Hospital das Clínicas da Faculdade de Medicina da Universidade de São Paulo, by clinical evaluation, sleep questionnaire, 
biochemical blood analysis, echocardiography and sleep study (overnight portable respiratory monitoring). We used a cut-off value of 15 and 30 apneas and hypopneas per hour of recording in the sleep study for the diagnosis of obstructive sleep apnea and severe obstructive sleep apnea, respectively. Results: We evaluated 80 consecutive patients with hypertrophic cardiomyopathy. Obstructive sleep apnea was present in 32 patients $(40 \%)$. Severe obstructive sleep apnea was present in 17 patients (21\%). Patients with obstructive sleep apnea were significantly older (56 [41-64] vs. 39 [30-53] years, $\mathrm{p}<0.001)$, presented higher body mass index $\left(28.2 \pm 3.5 \mathrm{vs} .25 .2 \pm 5.2 \mathrm{Kg} / \mathrm{m}^{2}, \mathrm{p}\right.$ $<0.01)$, increased left atrial diameter (45 [42-53] vs. 41 [39-47] mm, $\mathrm{p}=0.01)$ and aorta diameter (34 [30-37] vs. 29 [28-32] mm, p < 0.001) compared with patients without obstructive sleep apnea. Two models of stepwise multiple linear regression to identify variables associated with left atrial and ascending aorta enlargement showed that apnea-hypopnea index was the only variable associated with left atrial enlargement $(p=0.05)$ and aorta diameter $(p=0.01)$, respectively. Permanent atrial fibrillation was present in $31 \%$ vs. $6 \%$ in patients with and without obstructive sleep apnea, respectively $(p<0.01)$. Obstructive sleep apnea $(p=0.03)$ and left atrial diameter $(p=0.03)$ were the only factors independently associated with atrial fibrillation in a multivariate model. Conclusions: Obstructive sleep apnea is highly prevalent in patients with hypertrophic cardiomyopathy and it is associated with left atrial and ascending aorta enlargement. Obstructive sleep apnea is independently associated with atrial fibrillation, a risk factor for cardiovascular death in this population. 
Descriptors: 1. Obstructive sleep apnea; 2. Prevalence; 3. Hypertrophic cardiomyopathy; 4. Atrial fibrillation. 


\section{Introdução}


O presente trabalho aborda a prevalência dos distúrbios respiratórios do sono, bem como avalia a associação dos distúrbios respiratórios do sono com o remodelamento cardíaco e com a presença de fibrilação atrial (FA) em pacientes com miocardiopatia hipertrófica $(\mathrm{MH})$.

A introdução abaixo aborda tópicos que julgamos importantes para o contexto deste trabalho.

\subsection{Miocardiopatia Hipertrófica}

A MH é uma doença genética transmitida por herança autossômica dominante, caracterizada por hipertrofia ventricular esquerda de forma assimétrica, podendo gerar um gradiente de pressão na via de saída do ventrículo esquerdo. ${ }^{1,2,3} \mathrm{~A}$ MH é atualmente a principal causa de morte súbita em jovens, ${ }^{4}$ sendo a doença genética cardíaca mais prevalente, acometendo em torno de $0,16 \%$ da população geral. ${ }^{5} \mathrm{~A} \mathrm{MH}$ geralmente se manifesta na puberdade, porém, mutações após o nascimento podem ser responsáveis pelo acometimento em várias faixas etárias, variando da infância a fase adulta. De forma geral, os casos de apresentação mais precoces apresentam pior prognóstico. Entretanto, algumas mutações de aparecimento tardio também podem apresentar prognóstico adverso. ${ }^{6}$ Muitos pacientes com MH apresentamse assintomáticos ou com sintomas leves. Entretanto, naqueles referenciados 
INTRODUCÃ̃ 3

para centros terciários, sintomas de insuficiência cardíaca associados a gradientes de pressão na via de saída do ventrículo esquerdo são comuns. ${ }^{7} \mathrm{~A}$ dispnéia ao esforço é o sintoma mais comum, acometendo mais de $90 \%$ dos pacientes. A dispnéia pode resultar de uma variedade de mecanismos: disfunção diastólica secundária à hipertrofia miocárdica, impedimento ao esvaziamento ventricular secundário à obstrução, regurgitação mitral e, menos comumente, à disfunção sistólica. ${ }^{8}$

O diagnóstico da $\mathrm{MH}$ pode ser feito de forma fácil utilizando-se a ecocardiografia transtorácica, demonstrando-se hipertrofia ventricular esquerda de $15 \mathrm{~mm}$ ou mais, na ausência de outras doenças capazes de causar hipertrofia, ou espessura de 13 - $15 \mathrm{~mm}$, acompanhada de características típicas de $\mathrm{MH}$ e ausência de outras doenças capazes de causar hipertrofia. ${ }^{9}$

A mortalidade anual dos portadores de $\mathrm{MH}$ varia de 1 a $6 \% .^{10,11,12}$ Os fatores de risco para mortalidade cardiovascular em pacientes com $\mathrm{MH}$ estão resumidos na Tabela $1 .^{13}$ A FA é a arritmia sustentada mais comum em indivíduos com MH. A FA é um determinante independente de morbidade e mortalidade por insuficiência cardíaca e acidente vascular cerebral na MH. ${ }^{14,15,16}$ Recentemente, o aumento do átrio esquerdo também tem sido associado com risco aumentado de morte relacionada com insuficiência cardíaca em pacientes com MH. ${ }^{17,18}$ 
INTRODUÇÃO 4

Tabela 1: Fatores de risco para morte súbita na miocardiopatia hipertrófica.

Fatores de risco maiores

Fatores possíveis em casos específicos

Fibrilação ventricular / taquicardia

ventricular sustentada

História familiar de morte súbita

Isquemia miocárdica

Síncope inexplicada

Obstrução da via de saída do ventrículo esquerdo

Espessura ventricular $>30 \mathrm{~mm}$

Mutações de alto risco

Curva anormal de pressão arterial ao exercício

Atividade física intensa (competitiva)

Taquicardia ventricular não

sustentada ao Holter 
O tratamento da $\mathrm{MH}$ consiste em uso de terapias que reduzam a frequência cardíaca e o gradiente intraventricular. Beta-bloqueadores e bloqueadores do canal de cálcio são bastante utilizados. ${ }^{3}$ As arritmias ventriculares, comuns nesses pacientes, são tratadas com 0 uso de antiarrítmicos como a amiodarona ou disopiramida. O tratamento da FA baseiase no controle da frequência ventricular ou com reversão ao ritmo sinusal em casos selecionados, associando-se o uso de anticoagulação oral. Casos refratários ao tratamento clínico da insuficiência cardíaca podem se beneficiar de terapia invasiva para redução do septo interventricular. A alcoolização do septo por cateterismo intervencionista, bem como a miectomia cirúrgica são os procedimentos disponíveis até o momento. ${ }^{13,19} \mathrm{O}$ implante de cardioversordesfibrilador é atualmente indicado em casos de alto risco de morte súbita ou naqueles sobreviventes de arritmias graves. ${ }^{9}$ Nesse contexto, é importante 0 reconhecimento de novos fatores que possam estar associados à FA e ao remodelamento cardíaco em pacientes com $\mathrm{MH}$, já que a $\mathrm{MH}$ continua sendo uma causa importante de mortalidade cardiovascular precoce. 
INTRODUÇÃO 6

\subsection{Distúrbios respiratórios do sono}

O sistema respiratório e o sistema cardiovascular são responsáveis em conjunto para a realização das trocas gasosas. Ambos devem estar intimamente integrados para suprir as necessidades dos tecidos em resposta a diferentes demandas. Alterações em qualquer um dos sistemas geralmente levam a repercussões no outro. ${ }^{20}$ Esta complexa interação pode ser vista claramente nos pacientes com distúrbios respiratórios do sono, cada vez mais reconhecidos na população geral e caracterizados por pausas respiratórias recorrentes durante o sono.

As pausas respiratórias durante o sono podem ser classificadas em obstrutivas, na qual há esforço respiratório durante a pausa (Figura 1); centrais, na qual não existe esforço dos músculos durante a pausa (Figura 2) e mistas, que se caracterizam por iniciar como central (sem esforço) e terminar como obstrutiva, isto é, com esforço respiratório. ${ }^{21}$

\subsubsection{Apneia obstrutiva do sono (AOS)}

A AOS caracteriza-se por episódios repetidos de obstrução parcial ou total das vias aéreas superiores durante o sono e queda de saturação. ${ }^{22}$ A AOS é mais frequente em homens (24\%) do que em mulheres $(9 \%)$, com tendência ao aumento com o avanço da idade. ${ }^{23}$ 
Figura 1. Registro de polissonografia de um paciente com apneia obstrutiva do sono.

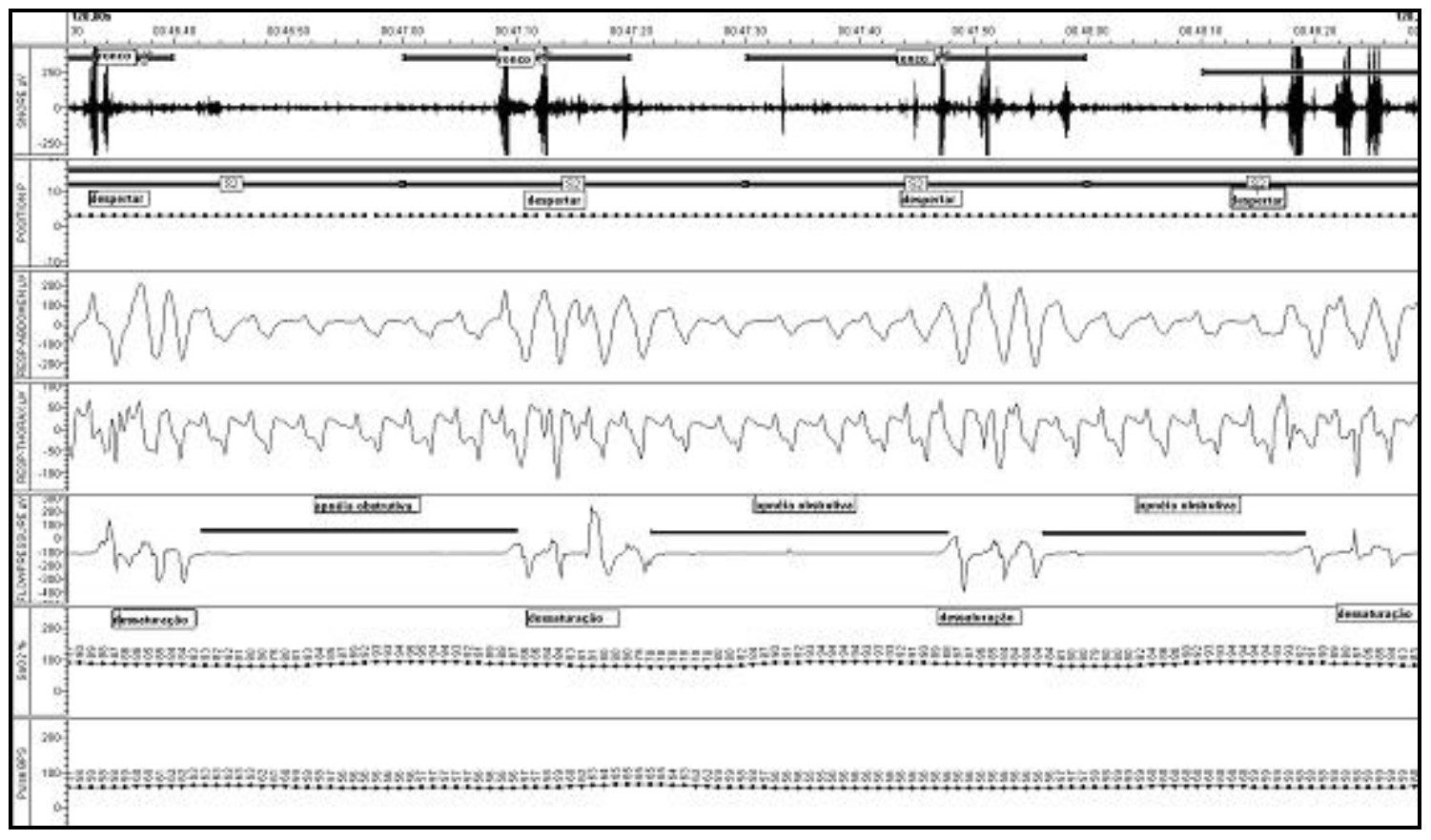

De cima para baixo: canal de ronco, estágio do sono, cinta torácica e abdominal, fluxo aéreo nasal, saturação de oxigênio e frequência cardíaca. Observar a cessação do fluxo aéreo nasal de forma recorrente (apneias) com manutenção do esforço tóraco-abdominal. Gravação de dois minutos de duração.

Uma das características clínicas marcantes da AOS é o ronco alto e constante durante 0 sono. ${ }^{24}$ A fragmentação do sono, causada por microdespertares frequentes, leva a muitos sintomas que incluem a hipersonolência diurna, fadiga crônica, irritabilidade, perda da libido, cefaleia matinal, perda de memória e de qualidade de vida. ${ }^{25}$ 
O exame de escolha para o diagnóstico dos distúrbios do sono é a polissonografia noturna, que apresenta sensibilidade e especificidade próximas de $95 \% .^{22}$ A polissonografia consiste na monitorização simultânea do eletroencefalograma, eletrooculograma, eletromiograma, saturação de oxigênio, fluxo de ar, movimentos torácicos e abdominais e frequência cardíaca. Desta forma, a polissonografia noturna permite não só a avaliação da arquitetura e a eficiência do sono, mas também diagnosticar e diferenciar os distúrbios do sono.

Como alternativa à polissonografia, monitores portáteis do sono com número limitado de canais (fluxo de ar, detecção de movimentos respiratórios, saturação periférica de oxigênio e frequência cardíaca) são bastante utilizados na prática clínica, permitindo um diagnóstico mais rápido e econômico dos distúrbios do sono em populações com alta probabilidade de doenças do sono. ${ }^{26}$

Os potenciais efeitos da AOS sobre o sistema cardiovascular são múltiplos. As apneias e/ou hipopneias recorrentes causam hipóxia; redução na pressão intratorácica com consequente aumento da pós-carga do ventrículo esquerdo pelo aumento da pressão transmural do ventrículo esquerdo (diferença entre a pressão intracardíaca e a pressão intratorácica) e aumento do retorno venoso com distensão do ventrículo direito e deslocamento do septo interventricular para a esquerda, o que leva a uma diminuição do enchimento ventricular; aumento da atividade nervosa simpática por estimulação dos quimiorreceptores centrais e periféricos desencadeado pela hipóxia e 
hipercapnia. ${ }^{21,27}$ Cabe destacar que estes fenômenos ocorrem centenas de vezes por noite e cronicamente podem contribuir para o remodelamento cardíaco.

A prevalência de AOS é muito maior em pacientes com doença cardiovascular estabelecida. ${ }^{28}$ Por exemplo, $50 \%$ dos pacientes com FA têm AOS. ${ }^{29}$ Além disso, a AOS não tratada tem sido associada com um risco 2 a 3 vezes maior de recorrência de FA após cardioversão elétrica comparado com o risco em pacientes tratados para AOS. ${ }^{29}$ AOS também tem sido associada com remodelamento atrial, ${ }^{30,31}$ que por sua vez, desempenha um papel importante no desenvolvimento de FA. ${ }^{32}$ Particularmente na hipertensão arterial sistêmica, estudos mostram uma prevalência de SAOS em 35\% dos hipertensos, chegando a $70 \%$ em casos de hipertensão arterial refratária. ${ }^{33}$ Já em pacientes com doença coronariana, a prevalência da AOS chega a 30\%. ${ }^{34}$

O conjunto de alterações cardiovasculares promovidos pela AOS pode ser, do ponto de vista teórico, especialmente deletério em pacientes com $\mathrm{MH}$, contribuindo para o surgimento de arritmias ou influindo negativamente no remodelamento ventricular.

O tratamento de escolha da AOS consiste no uso da pressão contínua positiva de vias aéreas superiores (CPAP). O CPAP é um aparelho que fornece um fluxo de ar por uma máscara facial ou nasal agindo como uma prótese pneumática para manter aberta a via aérea durante a inspiração e expiração. ${ }^{35}$ 
Assim, promove remissão dos eventos respiratórios e consequentemente melhora a estrutura do sono e a qualidade de vida dos pacientes.

O CPAP tem efeitos hemodinâmicos tanto na diminuição da pré-carga, como na pós-carga, eliminando as elevações da pressão arterial ${ }^{36} \mathrm{e}$ aumentando a pressão intratorácica, ambas responsáveis pela redução da póscarga durante o sono, além de reduzir a frequência cardíaca. ${ }^{37}$ Por outro lado, o uso agudo de CPAP em pacientes com insuficiência cardíaca estável e AOS diminuiu agudamente o volume sistólico e a fração de ejeção do ventrículo esquerdo. ${ }^{38}$ Essas alterações do CPAP na hemodinâmica agem, de uma forma geral, melhorando o desempenho cardíaco em pacientes com insuficiência cardíaca sistólica.

Até o momento, são escassos os estudos que avaliaram a AOS em pacientes com MH. Banno et al. ${ }^{39}$ avaliaram a AOS em 15 pacientes portadores de $\mathrm{MH}$, numa população de 35 pacientes com cardiopatias de várias etiologias. Os autores encontraram AOS em metade dos pacientes, sugerindo uma alta prevalência de AOS nessa população. Recentemente, Sengupta et al. ${ }^{40}$ relataram 4 pacientes com AOS e $\mathrm{MH}$ na forma obstrutiva com insuficiência cardíaca refratária encaminhados para cirurgia de miectomia, que melhoraram clinica e funcionalmente de forma importante com o uso de CPAP por longo prazo, evitando a cirurgia. 


\subsubsection{Apneia central do sono e Respiração Cheyne-Stokes}

Pacientes com insuficiência cardíaca sistólica podem apresentar um padrão de respiração periódica onde as apneias e hipopneias centrais alternamse com períodos de ventilação com um padrão crescente e posteriormente decrescente de volume corrente associados a despertares coincidentes com o pico de ventilação conhecida como Respiração de Cheyne-Stokes (RCSACS). ${ }^{41,42}$

A RCS-ACS leva a um sono fragmentado, ${ }^{43}$ oscilações da pressão arterial e do ritmo cardíaco, ${ }^{44,45,46}$ além de sintomas semelhantes à AOS, com sono de má qualidade, de arquitetura alterada e não restaurador, associados à hipersonolência e fadiga diurna. ${ }^{47,48}$ A presença de RCS-ACS em pacientes com insuficiência cardíaca sistólica está associada a risco aumentado de morte. ${ }^{49} \mathrm{~A}$ figura 2 representa um padrão polissonográfico típico de RCS-ACS. 
Figura 2. Registro de polissonografia de um paciente com Respiração de Cheyne-Stokes.

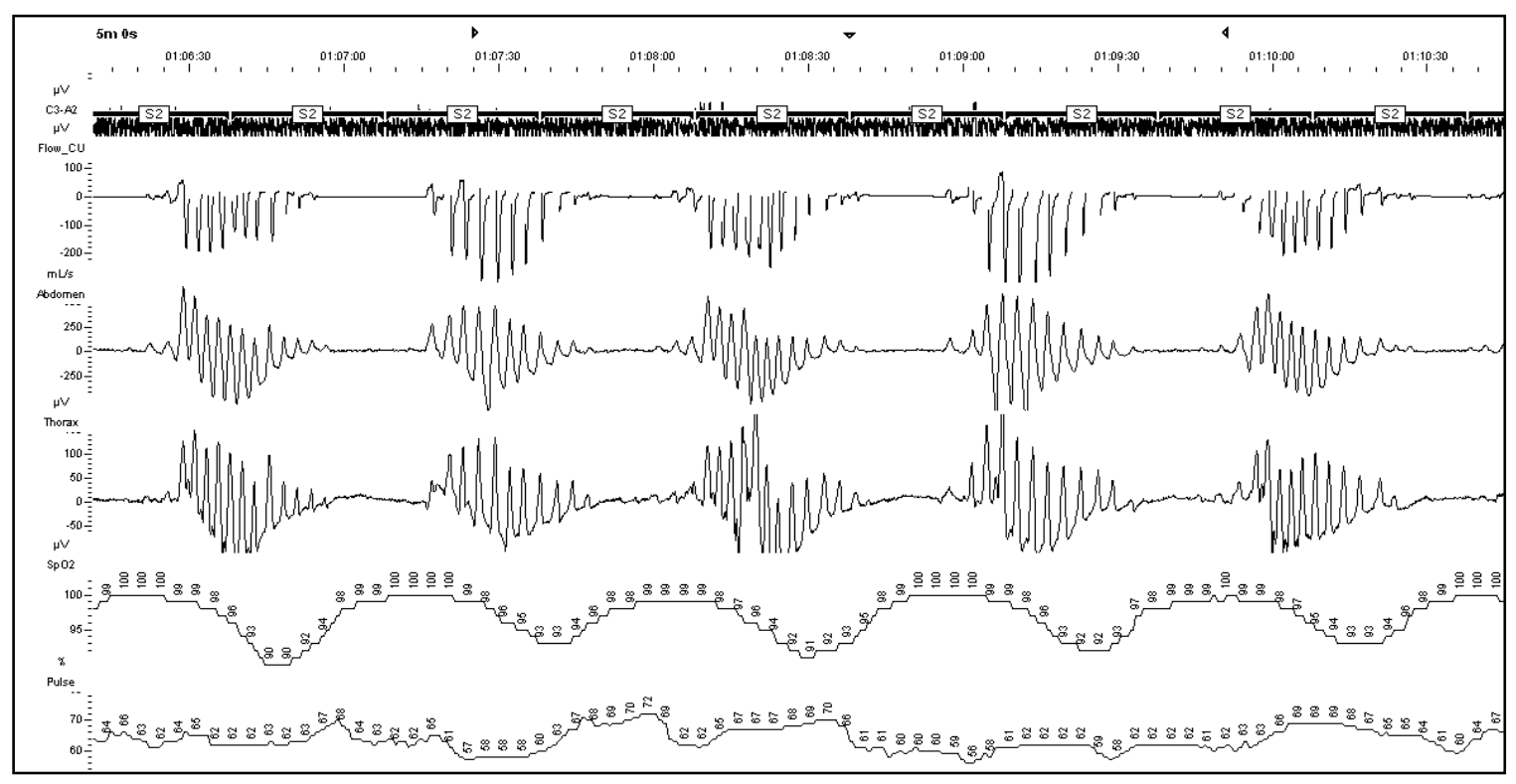

De cima para baixo: canal de eletroencefalograma, fluxo aéreo nasal, cinta torácica e abdominal, saturação de oxigênio e frequência cardíaca. Observar a cessação do fluxo aéreo nasal de forma recorrente (apneias) com ausência do esforço tóraco-abdominal. Gravação de cinco minutos de duração. 
2 RACIONAL DO ESTUDO 
Os distúrbios respiratórios do sono são muito prevalentes na população em geral, ${ }^{50}$ com uma prevalência ainda maior entre os pacientes com doença cardiovascular estabelecida. AOS está presente em $50 \%$ dos pacientes com fibrilação atrial| ${ }^{29}$, em um terço dos pacientes com doença coronariana ${ }^{34}$ e em até $70 \%$ dos pacientes com hipertensão resistente ${ }^{33}$. No presente trabalho fizemos a hipótese de que os distúrbios respiratórios do sono são comuns entre os pacientes portadores de MH. Fizemos também a hipótese de que a AOS tem influência negativa no sistema cardiovascular e estará associada de forma independente com remodelamento cardíaco, bem como com a presença de fibrilação atrial. 
3 OBJETIVOS 
- Avaliar a prevalência dos distúrbios respiratórios do sono em pacientes consecutivos acompanhados ambulatorialmente portadores de $\mathrm{MH}$.

- Avaliar a associação entre AOS com alterações estruturais cardíacas (ventricular e atrial) nos portadores de $\mathrm{MH}$.

- Avaliar a associação entre AOS com a presença de fibrilação atrial em portadores de $\mathrm{MH}$. 
3 CASUÍSTICA E MÉTODOS 


\subsection{População estudada}

O estudo foi conduzido no Laboratório do Sono da Divisão de Pneumologia do Instituto do Coração (InCor) do Hospital das Clínicas da Faculdade de Medicina da Universidade de São Paulo (HC-FMUSP), em conjunto com a Unidade Clínica de Miocardiopatias do InCor do HC-FMUSP. O protocolo foi aprovado pelo Comitê de Ética do InCor sob o número 0213/09 e os pacientes assinaram o termo de consentimento livre e esclarecido (ANEXO A). O período de recrutamento foi de março de 2008 a março de 2009.

Foram estudados de forma consecutiva todos os pacientes com $\mathrm{MH}$ recrutados do ambulatório da Unidade Clínica de Miocardiopatias do InCor.

Inclusão:

- indivíduos maiores de 18 anos;

- Pacientes que aceitaram participar do protocolo e assinaram o termo de consentimento.

Exclusão:

- pacientes instáveis do ponto de vista cardiovascular (mudança da medicação, da classe funcional de insuficiência cardíaca ou hospitalização nos últimos 30 dias); 
- pacientes submetidos à cirurgia cardíaca ou alcoolização percutânea do septo interventricular previamente;

- diagnóstico prévio de distúrbios respiratórios do sono;

- outras cardiopatias que alteram a morfologia cardíaca, como valvulopatias primárias;

- pacientes que realizaram estudo do sono de má qualidade.

\subsection{Avaliação clínica}

Todos os pacientes foram submetidos a uma avaliação clínica para medida de pressão arterial, peso, altura, índice de massa corpórea (IMC) e circunferência cervical. Os pacientes foram avaliados e classificados de acordo com a classe funcional da New York Heart Association (NYHA). Formam realizadas 3 mediadas da pressão arterial durante a consulta, utilizando-se um esfigmomanômetro de coluna de mercúrio, após o repouso por no mínimo 5 minutos. Utilizou-se a média das pressões das duas últimas aferições.

\subsection{Questionário}

A Escala de Sonolência de Epworth foi utilizada para avaliar a sonolência diurna subjetiva. Resumidamente, o paciente responde a oito perguntas que avaliam sua chance de cochilar em situações habituais do cotidiano, pontuando 
de 0 até 3 . Uma pontuação acima de 10 pontos foi considerada como presença de sonolência diurna excessiva. ${ }^{51}$

\subsection{Bioquímica e eletrocardiograma}

Os pacientes realizaram rotineiramente exames bioquímicos e hematimétricos gerais incluindo glicose de jejum, colesterol total e frações, triglicerídeos, hemoglobina e creatinina, além de eletrocardiograma de 12 derivações. Esses dados eram coletados no dia da consulta ambulatorial. Considerou-se apenas a presença de FA permanente para comparação entre os grupos estudados.

\subsection{Monitorização cardiorespiratória noturna}

Todos os pacientes foram monitorizados por uma noite com o monitor respiratório portátil StarDust II (Respironics, Inc, Murrysville, PA). Os pacientes receberam instruções do funcionamento do aparelho no laboratório do Sono do InCor e levaram o monitor portátil para casa. Todos foram instruídos a ligar o aparelho na hora de dormir e desligar ao acordar pela manhã. O monitor portátil monitoriza continuamente: oximetria, cinta tóraco-abdominal para detecção de esforço respiratório, fluxo aéreo medido por cânula nasal de pressão, detector de posição, captura de parâmetros de frequência cardíaca provindo da oximetria de pulso e detecção de ronco, tendo sido validado contra polissonografia 
completa em uma população referenciada para laboratório do sono.$^{52} \mathrm{~A}$ leitura do monitor portátil foi realizada no Laboratório do Sono em software próprio por um único observador experiente, que era cego quanto à queixas sugestivas de AOS. A leitura foi realizada manualmente, seguindo as normas internacionais. Apneia foi definida quando houve redução de mais de $90 \%$ do fluxo aéreo. Da mesma forma, hipopneia foi diagnosticada quando da redução de $50 \%$ do fluxo, associada à queda da saturação de $3 \%$ da oxi-hemoglobina..$^{53} \mathrm{O}$ tempo total em cama foi utilizado para a determinação do índice de apneia e hipopneia (IAH). O resultado final foi expresso em $\mathrm{IAH}$, saturação de oxi-hemoglobina mínima e porcentagem do tempo com $\mathrm{SaO}_{2}$ abaixo de $90 \% .{ }^{53} \mathrm{AOS}$ foi definida como um $\mathrm{IAH} \geq 15$ eventos/hora. AOS grave foi definida como um $\mathrm{IAH} \geq 30$ eventos/hora.

\subsection{Ecocardiograma}

O ecocardiograma bidimensional utilizando o modo-M foi obtido de acordo com as diretrizes da Sociedade Americana de Ecocardiografia. ${ }^{54}$ Os gradientes sistólicos de repouso foram medidos com ajuda do Doppler contínuo em toda a cavidade e da via de saída do ventrículo esquerdo, orientando o transdutor medialmente e anteriormente e evitando o jato de regurgitação mitral, quando presente. Os pacientes mantinham uma respiração normal e a velocidade de pico e o gradiente médio eram obtidos e registrados. O fluxo de entrada da válvula mitral foi registrado posicionando-se o cursor no nível da ponta do folheto valvar. A fração de ejeção do ventrículo esquerdo foi calculada pela fórmula de 
Simpson modificada. Os exames foram realizados com o aparelho Acuson comercialmente disponível (Sequoia) equipado com transdutores de 2,5 e 3,5 $\mathrm{MHz}$ por um único observador experiente, que era cego quanto à presença ou ausência de AOS, com um intervalo do estudo do sono não superior a seis meses. Os diâmetros internos (diastólico e sistólico) finais do ventrículo esquerdo, as espessuras diastólicas da parede posterior e do septo interventricular do ventrículo esquerdo e o diâmetro atrial esquerdo foram registrados. Padrões de enchimento diastólico foram classificados com base em estudos prévios. ${ }^{55}$ Todos os pacientes preencheram os critérios para diagnóstico de $\mathrm{MH}$, definidos por uma espessura mínima da parede ventricular esquerda de $15 \mathrm{~mm}$ em qualquer segmento na ausência de causas conhecidas de hipertrofia ventricular esquerda. ${ }^{9}$

\subsection{Análise dos dados}

Os resultados foram expressos como média \pm desvio padrão para variáveis contínuas com distribuição normal ou mediana (intervalo interquartil) para variáveis contínuas com distribuição não normal. O Teste T de Student ou o Teste de Mann-Whitney para amostras independentes foram utilizados para comparação de variáveis contínuas entre grupos com distribuição normal e não normal, respectivamente. O Teste do Qui-quadrado foi utilizado para comparar as variáveis categóricas entre os pacientes com e sem AOS, quando apropriado. Dois modelos de regressão linear múltipla do tipo stepwise foram utilizados para 
avaliar as variáveis clínicas, antropométricas e ecocardiográficas associadas com diâmetros do átrio esquerdo e da aorta ascendente. As variáveis independentes nesses modelos foram: idade, hipertensão arterial, índice de massa corporal (IMC), IAH. A variável categórica gradiente de saída do ventrículo esquerdo maior do que $30 \mathrm{~mm} \mathrm{Hg}$ e a fração de ejeção do ventrículo esquerdo também foram incluídas como variáveis no modelo do diâmetro atrial esquerdo, especificamente. Um modelo de regressão logística foi utilizado para determinar os fatores associados à FA. As variáveis independentes incluídas na análise univariada foram: idade, classe funcional, hipertensão arterial, IMC, IAH, saturação mínima de oxi-hemoglobina, história de síncope e variáveis ecocardiográficas. Variáveis com o $\mathrm{P}<0,1$ na análise univariada foram inseridas em uma análise multivariada. O nível de significância foi fixado em $P<0,05$. Os cálculos foram realizados utilizando o Minitab® Release 15 Statistical Software (State College, PA, E.U.A.). 
5RESULTADOS 
Foram avaliados prospectivamente 92 pacientes com $\mathrm{MH}$. Doze pacientes foram excluídos por causa de um ou mais critérios de exclusão e, portanto, 80 pacientes foram estudados (Figura 3).

Figura 3. Diagrama de fluxo de pacientes

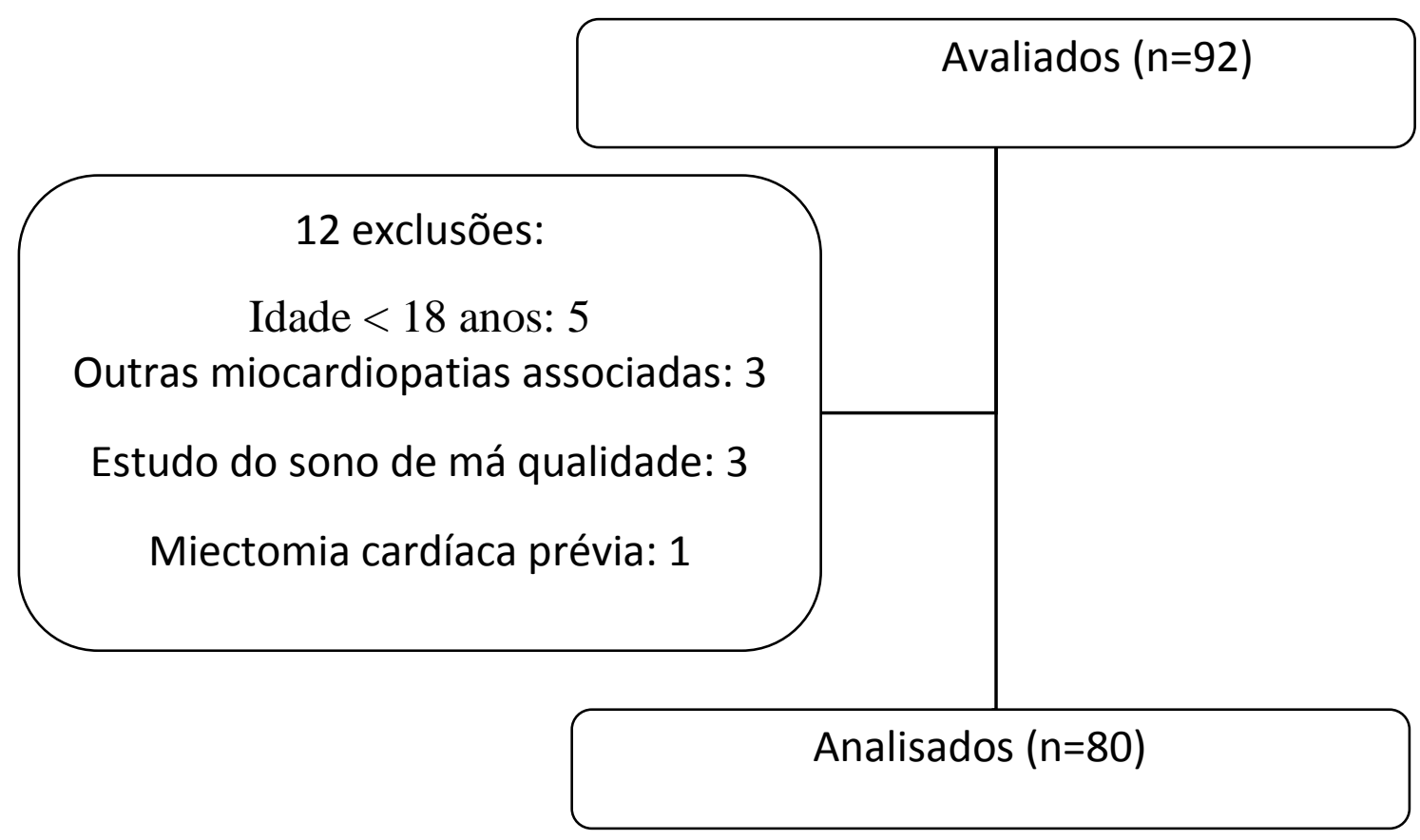


As características basais da população geral do estudo, bem como as características dos indivíduos divididos de acordo com a ausência ou presença de AOS são descritas na Tabela 2, 3 e 4. AOS foi diagnosticada em 32 pacientes (40\%), 17 pacientes (21\%) apresentavam AOS grave. Pacientes com e sem AOS foram semelhantes quanto a sexo, raça, pressão arterial, frequência cardíaca, glicemia de jejum, colesterol total e frações, hemoglobina, creatinina e sonolência diurna. Em contraste, pacientes com AOS eram mais velhos, tinham um IMC mais elevado, maior circunferência da cintura e do pescoço, e níveis de triglicerídeos mais elevados (Tabela 2 e 3). Um padrão de RCS-ACS foi identificado em 7 pacientes (9\%). No entanto, quando retirados os eventos centrais da análise, todos esses indivíduos apresentavam predominantemente eventos obstrutivos (IAH > 15 eventos/hora) e então foram considerados como apresentando AOS.

As características ecocardiográficas em pacientes com e sem AOS foram semelhantes em relação à fração de ejeção do ventrículo esquerdo $(72,2 \pm 11,4$ vs. $68,5 \pm 8,5 \%, \mathrm{P}=0,10)$, espessura do septo interventricular $(21,5[18,2-27,5]$ vs. $20,5[18,2-23,8] \mathrm{mm}, \mathrm{P}=0,23)$, a espessura da parede posterior $(12,5[11$ 12,5] vs. 11,0 [10-14] mm, $\mathrm{P}=0,41$ ) e diâmetro diastólico do ventrículo esquerdo (41 [38-46] vs. $43[40-47] \mathrm{mm}, \mathrm{P}=0,08)$. O percentual de pacientes com e sem AOS que apresentaram obstrução da via de saída do ventrículo esquerdo (> 30 $\mathrm{mm} \mathrm{Hg}$ de gradiente) também foi semelhante (38 vs. $31 \%, \mathrm{P}=0,57$ ). 
Tabela 2. Características clínicas, bioquímicas e medicações da população estudada total e segundo a presença ou ausência de AOS.

\begin{tabular}{|c|c|c|c|c|}
\hline & $\begin{array}{l}\text { Total } \\
\mathrm{N}=80\end{array}$ & $\begin{array}{c}\text { Sem AOS } \\
\quad N=48\end{array}$ & $\begin{array}{l}\text { AOS } \\
N=32\end{array}$ & $\begin{array}{l}\text { Valor } \\
\text { de } P\end{array}$ \\
\hline Idade, anos & $47(32-58)$ & $38,5(30-53)$ & $56(41-64)$ & 0,0001 \\
\hline Homens, \% & 49 & 46 & 53 & 0,52 \\
\hline Raça branca, \% & 76 & 79 & 72 & 0,45 \\
\hline Tabagismo, \% & 9 & 4 & 16 & 0,11 \\
\hline Índice de massa corpórea, $\mathrm{kg} / \mathrm{m}^{2}$ & $26,4 \pm 4,8$ & $25,2 \pm 5,2$ & $28,2 \pm 3,5$ & $<0,01$ \\
\hline Circunferência cervical, cm & $37,3 \pm 4,3$ & $36,1 \pm 3,1$ & $39,1 \pm 5,2$ & $<0,01$ \\
\hline Circunferência da cintura, cm & $93,6 \pm 12,1$ & $90,5 \pm 11,6$ & $98,2 \pm 11,5$ & $<0,01$ \\
\hline PA sistólica, mm Hg & $112(106-127)$ & $112(109-124)$ & $113(101-132)$ & 0,72 \\
\hline PA diastólica, mm Hg & $70(70-78)$ & $70(70-74)$ & $72(62-80)$ & 0,81 \\
\hline Frequência cardíaca, bpm & $64(60-70)$ & $64(60-70)$ & $60(56-71)$ & 0,24 \\
\hline CF IC NYHA III - IV, \% & 26 & 19 & 37 & 0,06 \\
\hline Tratamento clínico, \% & 88 & 83 & 94 & 0,17 \\
\hline Diurético, \% & 23 & 21 & 25 & 0,66 \\
\hline Beta-bloqueador, \% & 63 & 63 & 72 & 0,38 \\
\hline Bloqueador de Cálcio, \% & 23 & 19 & 29 & 0,33 \\
\hline Inibidor de ECA, \% & 4 & 2 & 6 & 0,56 \\
\hline Bloqueador de AT1, \% & 18 & 15 & 22 & 0,40 \\
\hline Amiodarona, \% & 16 & 15 & 19 & 0,62 \\
\hline
\end{tabular}


Valores expressos em média $( \pm D P)$. Variáveis com distribuição não normal são apresentadas com mediana (intervalo interquartil). AOS: apneia obstrutiva do sono; PA: pressão arterial; CF IC NYHA: classe funcional de insuficiência cardíaca da New York Heart Association; ECA: enzima conversora de angiotensina; AT1: angiotensina 1

A função diastólica não foi avaliada em 21 pacientes devido à presença de fibrilação atrial $(n=13)$ ou limitações técnicas $(n=8)$. Dentre os 59 pacientes restantes (40 sem e 19 com AOS), a prevalência de disfunção diastólica foi semelhante nos dois grupos ( $40 \%$ vs. $42 \%, P=0,87$; respectivamente). Em contraste, quando comparados a pacientes sem AOS, os pacientes com AOS apresentaram significativo aumento do diâmetro do átrio esquerdo e da aorta ascendente (41 [39-47] vs. 45 [42-52,8] mm, $\mathrm{P}=0,01),(29$ [28-32] vs. 34 [30-37] mm, P <0,001), respectivamente (Figura 4, 5). A única variável independentemente associada com o diâmetro atrial esquerdo e com o diâmetro da aorta ascendente nos dois modelos de regressão linear múltipla do tipo stepwise foi o IAH $\left(P=0,05 ; R^{2}\right.$ ajustado $\left.=11 \%\right)$ e $\left(P=0,01 ; R^{2}\right.$ ajustado $=$ $12,8 \%)$, respectivamente.

A FA foi significativamente mais comum em pacientes com (31\%) do que sem (6\%) AOS (Figura 6). O IAH nos pacientes com vs. sem FA foi de 32,9 (1755) vs. 7,9 (3-21) eventos/hora, respectivamente $(P<0,001)$. As variáveis 
associadas com FA determinadas pela regressão logística foram: idade, classe funcional da insuficiência cardíaca da NYHA, IAH, fração de ejeção do ventrículo esquerdo e diâmetro atrial esquerdo (Tabela 5). A análise multivariada mostrou que apenas 0 diâmetro do átrio esquerdo e 0 IAH foram fatores independentemente associados à FA (Tabela 6). 
Tabela 3. Características laboratoriais e ecocardiográficas da população estudada total e segundo a presença ou ausência de AOS.

\begin{tabular}{|c|c|c|c|c|}
\hline & $\begin{array}{l}\text { Total } \\
\mathrm{N}=80\end{array}$ & $\begin{array}{c}\text { Sem AOS } \\
\qquad N=48\end{array}$ & $\begin{array}{l}\text { AOS } \\
\mathrm{N}=32\end{array}$ & $\begin{array}{l}\text { Valor } \\
\text { de } P\end{array}$ \\
\hline \multicolumn{5}{|l|}{ Exames laboratoriais } \\
\hline Creatinina, mg/dL & $0,98(0,78-1,12)$ & $0,92(0,75-1,1)$ & $1,0(0,9-1,2)$ & 0,12 \\
\hline Glicemia de jejum, mg/dL & $92(86-101)$ & $92(86-98)$ & $95(89-105)$ & 0,16 \\
\hline Colesterol total, $\mathrm{mg} / \mathrm{dL}$ & $182 \pm 47$ & $185 \pm 49$ & $178 \pm 45$ & 0,59 \\
\hline Colesterol LDL, mg/dL & $115 \pm 37$ & $116 \pm 41$ & $114 \pm 33$ & 0,79 \\
\hline Colesterol HDL, mg/dL & $39(33-46)$ & $44(38-51)$ & $44(31-44)$ & 1,00 \\
\hline Hemoglobina, g/dL & $14,1(13,4-15,3)$ & $14,1(13,5-15,4)$ & $14,1(13-15,2)$ & 0,60 \\
\hline Triglicerídeos, mg/dL & $103(72-150)$ & $79(47-145)$ & $131(96-178)$ & $<0,01$ \\
\hline \multicolumn{5}{|l|}{ Ecocardiograma } \\
\hline Fração de ejeção, \% & $70,7 \pm 10,4$ & $68,5 \pm 8,5$ & $72,2 \pm 11,4$ & 0,10 \\
\hline Septo interventricular, $\mathrm{mm}$ & $21(18-25)$ & $20,5(18-24)$ & $21,5(18-28)$ & 0,23 \\
\hline Parede posterior, mm & $12(10-14)$ & $11(10-14)$ & $12,5(11-13)$ & 0,41 \\
\hline Diâmetro diastólico, mm & $42,5(39-47)$ & $43(40-47)$ & $41(38-46)$ & 0,08 \\
\hline Átrio esquerdo, mm & $42,5(39-49)$ & $41(38-46)$ & $45(42-53)$ & 0,01 \\
\hline Aorta, mm & $31(29-34)$ & $29(28-32)$ & $34(30-37)$ & 0,001 \\
\hline Obstrução da VSVE, \% & 34 & 31 & 38 & 0,57 \\
\hline Função diastólica alterada, \% & 41 & 42 & 40 & 0,87 \\
\hline
\end{tabular}


Valores expressos em média $( \pm D P)$. Variáveis com distribuição não normal são apresentadas com mediana (intervalo interquartil). AOS: apneia obstrutiva do sono; VSVE: via de saída do ventrículo Esquerdo 
Tabela 4. Exame do sono da população estudada total e segundo a presença ou ausência de AOS.

\begin{tabular}{lcccr}
\hline & $\begin{array}{c}\text { Total } \\
\mathrm{N}=80\end{array}$ & $\begin{array}{c}\text { Sem AOS } \\
\mathrm{N}=48\end{array}$ & $\begin{array}{c}\text { AOS } \\
\mathrm{N}=32\end{array}$ & $\begin{array}{c}\text { Valor } \\
\text { de P }\end{array}$ \\
\hline Escala de Epworth & $7(3-11)$ & $7(3-11)$ & $7(3-14)$ & 0,78 \\
Tempo de registro, min & $427 \pm 82$ & $417 \pm 83$ & $442 \pm 78$ & 0,19 \\
IAH, e/h & $9,2(4-25)$ & $5(2-8)$ & $30(22-41)$ & $<0,001$ \\
\multicolumn{1}{c}{ Apneia Central, e/h } & $0,3(0-1)$ & $0,2(0-1)$ & $1,3(0-5)$ & $<0,01$ \\
\multicolumn{1}{c}{ Apneia Obstrutiva, e/h } & $2,9(0,7-9,8)$ & $0,8(0-2)$ & $11,5(7-21)$ & $<0,01$ \\
\multicolumn{1}{c}{ Apneia mista, e/h } & $0(0-0,2)$ & $0(0-0,1)$ & $0,2(0-0,6)$ & $<0,01$ \\
\multicolumn{1}{c}{ Hipopneias, e/h } & $4,5(2-8)$ & $2,9(1-7)$ & $8,3(5-17)$ & $<0,01$ \\
Sat. mínima O $_{2}$ \% & $84(78-88)$ & $87(83-89)$ & $79(75-82)$ & $<0,001$ \\
\hline
\end{tabular}

Valores expressos em média ( $\pm \mathrm{DP})$. Variáveis com distribuição não normal são apresentadas com mediana (intervalo interquartil). AOS: apneia obstrutiva do sono; IAH: índice de apneia-hipopneia; e/h: eventos/hora; Sat $\mathrm{O}_{2}$ : saturação de oxi-hemoglobina. 
Figura 4. Diâmetro do átrio esquerdo em pacientes com miocardiopatia hipertrófica sem e com apneia obstrutiva do sono.

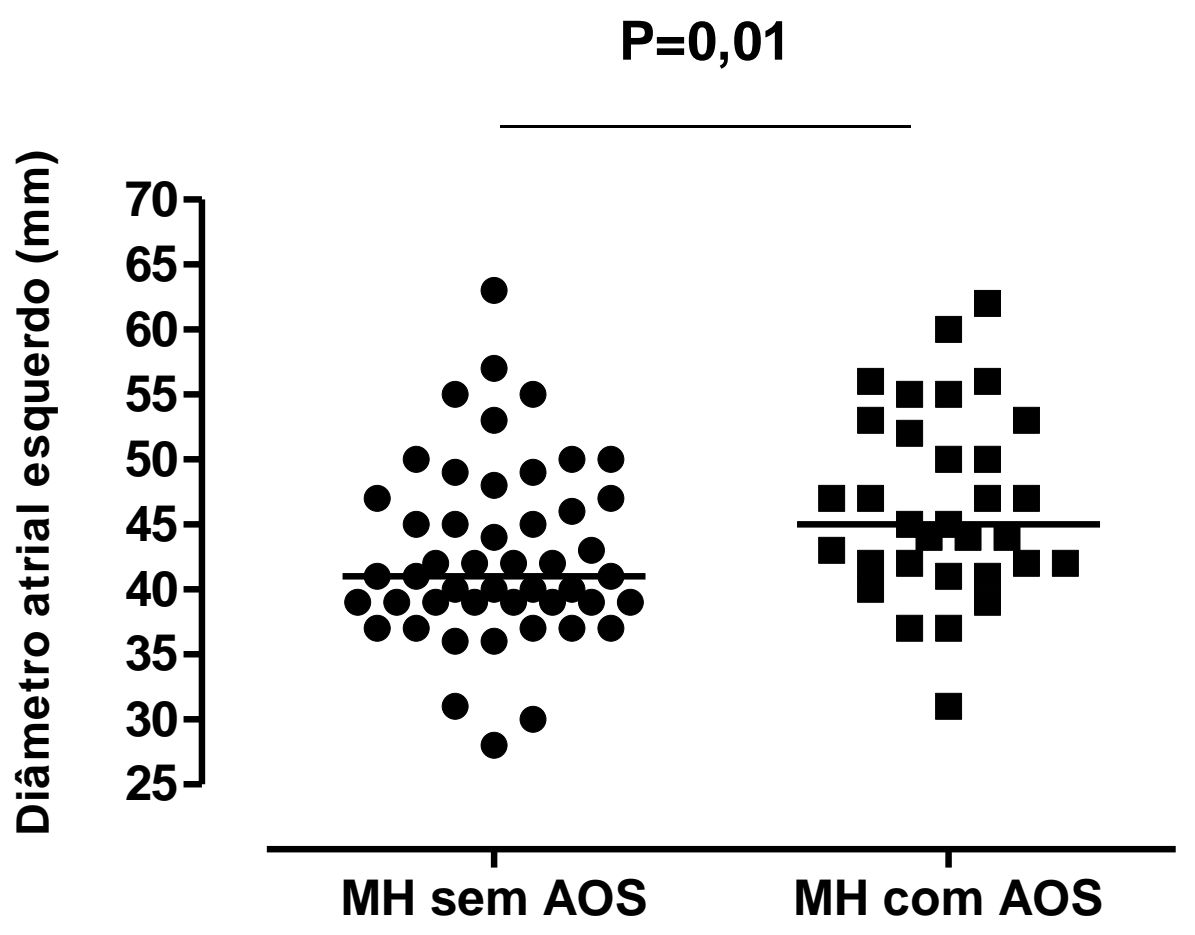


Figura 5. Diâmetro da aorta ascendente em pacientes com miocardiopatia hipertrófica sem e com apneia obstrutiva do sono.

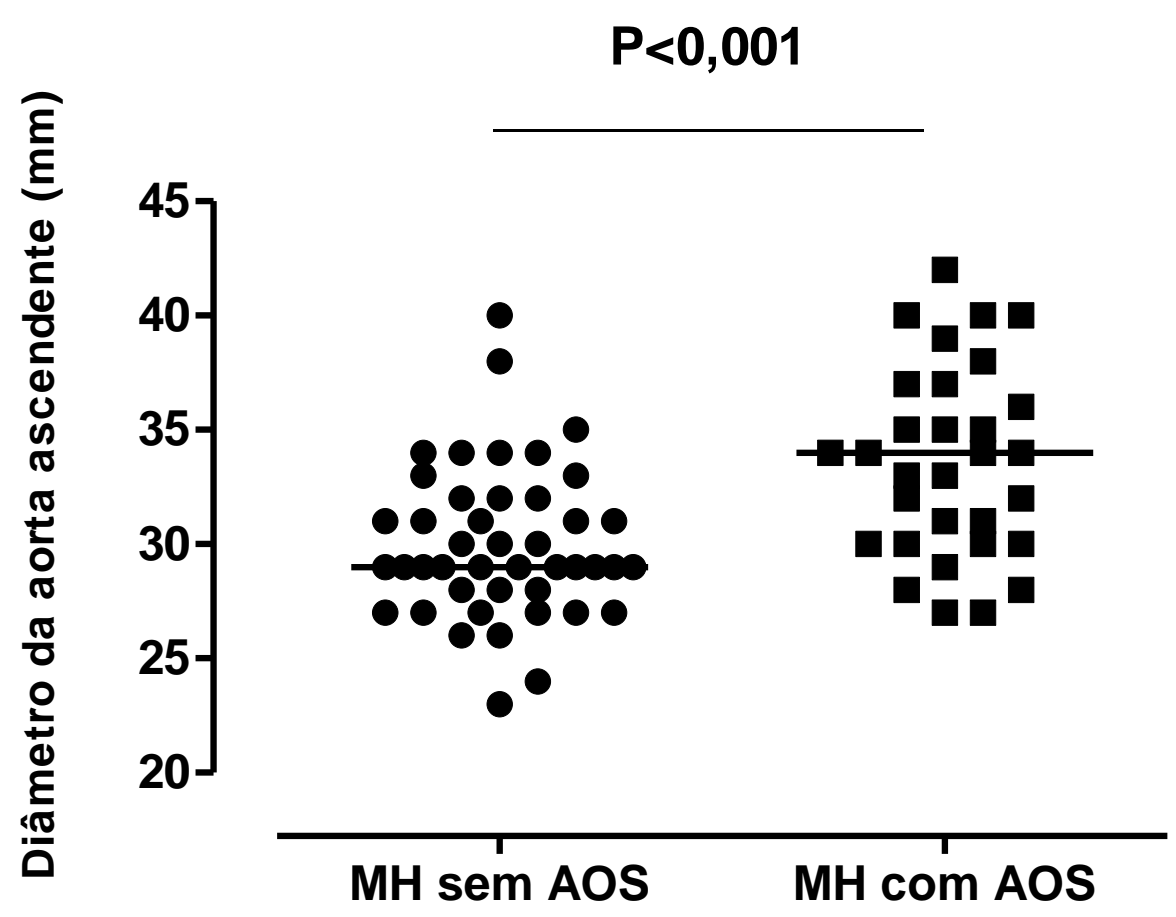


Figura 6. Presença de fibrilação atrial em pacientes com miocardiopatia hipertrófica sem e com apneia obstrutiva do sono.

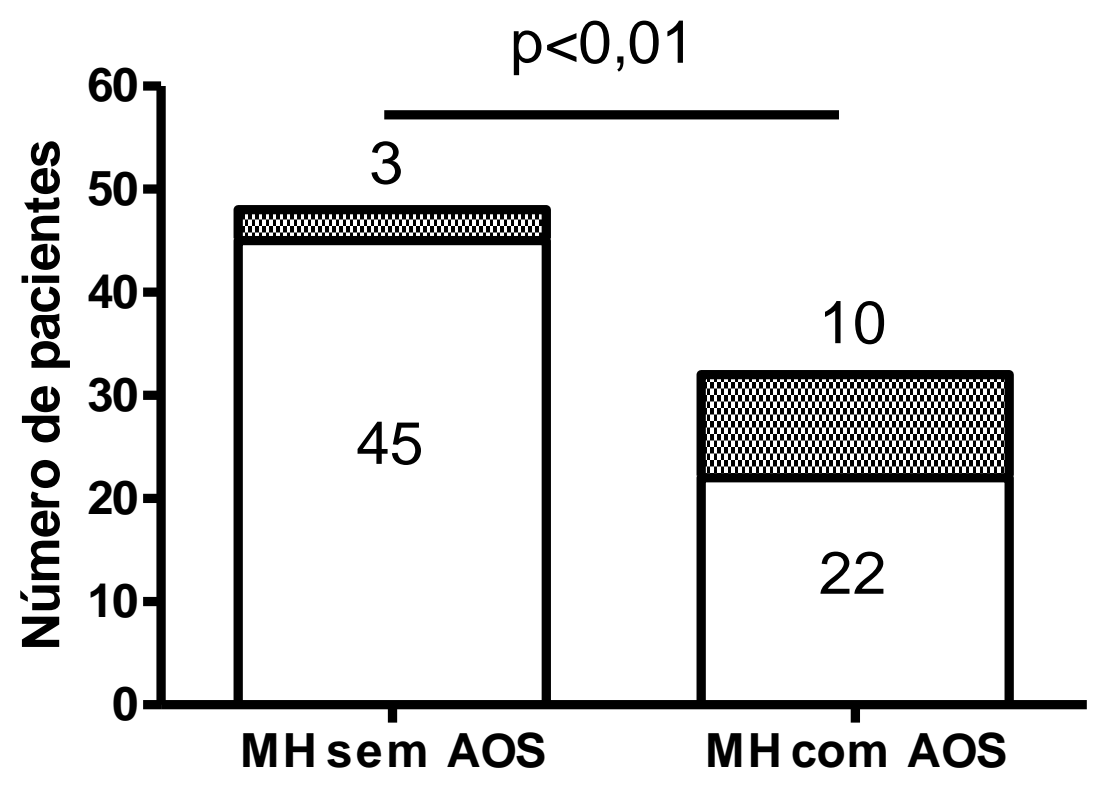

$\square$ Ritmo sinusal

Fibrilação atrial 
Tabela 5: Regressão logística entre a presença de fibrilação atrial com variáveis antropométricas, diagnóstico de hipertensão arterial, variáveis ecocardiográficas e do exame do sono.

\begin{tabular}{lccc}
\hline & $\begin{array}{c}\text { Razão de } \\
\text { chances }\end{array}$ & IC (95\%) & Valor de P \\
\hline Idade & 1,05 & $1,01-1,10$ & 0,03 \\
Classe funcional IC NYHA & 2,93 & $1,37-6,24$ & $<0,01$ \\
Hipertensão arterial & 2,52 & $0,74-8,55$ & 0,14 \\
Índice de massa corpórea & 1,07 & $0,94-1,21$ & 0,29 \\
Índice de apneia-hipopneia & 1,08 & $1,04-1,13$ & $<0,001$ \\
Saturação mínima de oxi-hemoglobina & 0,97 & $0,93-1,02$ & 0,25 \\
Síncope & 1,93 & $0,44-8,4$ & 0,38 \\
Septo interventricular > 30 mm & 0,38 & $0,05-3,23$ & 0,38 \\
Gradiente de VSVE > 30 mm Hg & 0,29 & $0,06-1,4$ & 0,12 \\
FE do ventrículo esquerdo & 0,95 & $0,9-1,01$ & 0,09 \\
Insuficiência mitral moderada/grave & 0,88 & $0,1-8,03$ & 0,90 \\
Diâmetro atrial esquerdo & 1,16 & $1,06-1,27$ & $<0,01$ \\
Diâmetro da aorta ascendente & 1,06 & $0,91-1,22$ & 0,48 \\
\hline
\end{tabular}

IC: intervalo de confiança; Classe funcional IC NYHA: classe funcional de insuficiência cardíaca da New York Heart Association; VSVE: via de saída do ventrículo esquerdo; FE: fração de ejeção. 
Tabela 6: Regressão logística múltipla entre a presença de fibrilação atrial com as variáveis significativas da análise univariada.

\begin{tabular}{lccc}
\hline & $\begin{array}{c}\text { Razão de } \\
\text { chances }\end{array}$ & IC (95\%) & Valor de P \\
\hline Idade & 1,02 & $0,94-1,11$ & 0,62 \\
Classe funcional IC NYHA & 1,88 & $0,67-5,24$ & 0,23 \\
Índice de apneia-hipopneia & 1,07 & $1,01-1,13$ & 0,03 \\
$\begin{array}{l}\text { Fração de ejeção do ventrículo } \\
\text { esquerdo }\end{array}$ & 0,94 & $0,88-1,01$ & 0,10 \\
Diâmetro atrial esquerdo & 1,17 & $1,02-1,34$ & 0,03 \\
\hline
\end{tabular}

IC: intervalo de confiança; Classe funcional IC NYHA: classe funcional de insuficiência cardíaca da New York Heart Association. 
6 DISCUSSÃO 
Esse estudo avaliou a presença de distúrbios respiratórios de sono em uma grande série de pacientes consecutivos com $\mathrm{MH}$ e contribui com os seguintes achados: em primeiro lugar, $40 \%$ dos pacientes com $\mathrm{MH}$ tinham $\mathrm{AOS}$ e apenas uma pequena proporção (9 \%) dos indivíduos apresentaram um padrão de RCS-ACS em associação à AOS. Em segundo lugar, os pacientes com AOS não apresentaram sinais de remodelamento cardíaco ventricular, mas sim de remodelamento atrial esquerdo, caracterizado pelo aumento do diâmetro atrial esquerdo. Em terceiro lugar, FA, um marcador de mortalidade cardíaca em pacientes com $\mathrm{MH},{ }^{16}$ foi 5 vezes mais comum em pacientes com AOS do que nos pacientes sem AOS. Finalmente, 0 diâmetro do átrio esquerdo e 0 diagnóstico de AOS foram os únicos fatores independentemente associados à FA na análise multivariada. Estes dados sugerem que a AOS possa contribuir para a morbidade cardiovascular observada em pacientes com $\mathrm{MH}$.

A prevalência da AOS na população de pacientes relatados neste estudo é extremamente elevada e pelo menos duas vezes superior que a encontrada na população geral. ${ }^{50}$ Durante o processo de submissão do artigo, um estudo independente reportou os resultados de oximetria noturna em 100 pacientes com $\mathrm{MH}$. Nesse estudo, encontrou-se uma prevalência de oximetria noturna sugestiva de AOS em $71 \%$ dos pacientes. Esse achado está em conformidade com nossos resultados, e sugere que a AOS é frequente nesses indivíduos. Outro achado interessante nesse mesmo estudo foi que uma maior proporção de pacientes com $\mathrm{MH}$ com oximetria alterada encontravam-se em classe 
funcional II ou III da NYHA em comparação com os pacientes sem oximetria alterada (83 vs. $62 \%, \mathrm{p}=0,02$; respectivamente) ${ }^{56} \mathrm{O}$ nosso trabalho portanto complementa esses dados, pois comprovou, de forma objetiva, uma alta prevalência de AOS na população de pacientes com MH. De forma semelhante, porém sem atingir significância estatística, em nosso estudo, os indivíduos com MH e AOS apresentavam-se mais frequentemente em CF III-IV da NYHA do que os indivíduos com $\mathrm{MH}$ sem AOS (37 vs. 19\%, $\mathrm{p}=0,06$; respectivamente). Algumas características dos pacientes com AOS estudados são importantes. Primeiramente, os pacientes com $\mathrm{MH}$ e AOS eram relativamente magros com um IMC ( $\left.28 \mathrm{~kg} / \mathrm{m}^{2}\right)$, que é menor do que o IMC em pacientes com AOS típica extraída da população geral $(\sim 35 \mathrm{~kg} / \mathrm{m} 2) \cdot{ }^{57}$ Este padrão tem sido previamente descrito em outras populações específicas, incluindo pacientes com insuficiência cardíaca congestiva ${ }^{58}$ e pacientes submetidos à hemodiálise. ${ }^{59}$ Uma possível explicação para essa alta prevalência de AOS em pacientes relativamente magros pode ser a redistribuição dos fluidos das pernas edemaciadas aos tecidos da faringe, quando se deslocam da posição vertical para supina durante a noite, interferindo no lúmen da faringe e predispondo ao colapso durante 0 sono. ${ }^{60,61}$ Outra característica destes pacientes com AOS é a falta de sonolência diurna excessiva, uma característica dos pacientes com AOS referidos a laboratórios do sono. A ausência de queixas de sono também tem sido descrita em outras populações de pacientes com doenças cardiovasculares, incluindo insuficiência cardíaca, ${ }^{58}$ síndrome metabólica, ${ }^{62,63}$ pacientes com indicação de 
marcapasso cardíaco, ${ }^{64}$ e portadores de hipertensão arterial sistêmica. ${ }^{65} \mathrm{~A}$ falta de sinais e sintomas típicos ligados à AOS nessa população pode ajudar a explicar a ausência de estudos prévios de avaliação sistemática do sono em pacientes com $\mathrm{MH}$. A ausência de diferença da pressão arterial entre os grupos sem e com AOS é curiosa, já que vários estudos populacionais têm associado a AOS com o risco de desenvolvimento de hipertensão arterial sistêmica. ${ }^{24}$ No entanto, a grande maioria dos indivíduos dos dois grupos usavam antihipertensivos para o controle dos sintomas relacionados à $\mathrm{MH}$, o que pode contribuir para o controle pressórico adequado dos dois grupos, além do fato do presente estudo não ter sido desenhado com esse intuito. Ainda nas características basais da população estudada, observamos que os pacientes do grupo AOS apresentavam maiores níveis de triglicerídeos comparados ao grupo sem AOS. Esse achado foi recentemente confirmado em pacientes consecutivos com síndrome metabólica. ${ }^{66}$ Estes dados sugerem que a AOS parece também influenciar o metabolismo lipídico, mas novos estudos são necessários para definir os mecanismos precisos envolvidos nesta associação. ${ }^{66}$

O pequeno número de indivíduos que apresentaram um padrão de RCSACS era esperado, já que os pacientes com MH não apresentavam disfunção sistólica do ventrículo esquerdo, presente na grande maioria dos pacientes com esse padrão respiratório. Recentemente, Pedrosa et al. ${ }^{67}$ descreveram o padrão de RCS-ACS em um paciente com MH, atribuindo a gênese da RCS-ACS à presença de disfunção diastólica do ventrículo esquerdo e à fibrilação atrial. 
A AOS tem sido associada previamente com o aumento da espessura do septo interventricular, diâmetro do átrio esquerdo e massa ventricular esquerda em pacientes hipertensos ${ }^{30}$ e com o aumento da massa ventricular esquerda e da espessura do septo interventricular em pacientes com insuficiência cardíaca congestiva ${ }^{68}$ Apneias obstrutivas provocam uma série de mudanças mecânicas, hemodinâmicas, químicas, neurais e inflamatórias com potenciais consequências adversas ao coração. ${ }^{21}$ Durante as apneias obstrutivas, é gerada uma pressão intratorácica negativa contra a via aérea superior fechada, o que aumenta a pressão transmural do ventrículo esquerdo, um importante determinante da pós-carga ventricular esquerda. Estas respostas mecânicas secundárias à AOS podem também ser importantes para o aumento da póscarga atrial esquerda, contribuindo para o aumento do diâmetro atrial esquerdo. ${ }^{31}$ Semelhantemente a estudos anteriores, encontramos um diâmetro atrial esquerdo aumentado em pacientes com MH e AOS em comparação com pacientes com $\mathrm{MH}$ sem AOS. No presente estudo, os pacientes com AOS possuíam um diâmetro atrial esquerdo que foi em média $9 \%$ maior do que pacientes sem AOS, que é notavelmente semelhante a um estudo anterior em pacientes com AOS, mas sem $\mathrm{MH}(9 \%) .{ }^{31}$ Outra constatação importante do nosso estudo é a observação de uma associação independente entre o diâmetro da aorta ascendente e AOS, mesmo após ajuste para fatores de confusão. Este resultado está de acordo com o estudo de Serizawa et al. ${ }^{69}$ em pacientes com AOS e sem cardiopatia significativa. O aumento da pressão transmural da 
parede da aorta, devido à pressão negativa intratorácica durante as apneias pode ter um papel central na dilatação da aorta. Ao contrário do que seria esperado a partir de estudos anteriores em pacientes com AOS, não houve diferença significativa no remodelamento ventricular esquerdo nos pacientes com e sem AOS. O número de pacientes com obstrução da via de saída do ventrículo esquerdo, definido como um gradiente maior do que $30 \mathrm{~mm} \mathrm{Hg}$, em pacientes com e sem AOS também foi semelhante. Nós especulamos que os determinantes genéticos da hipertrofia do ventrículo esquerdo que caracterizam a MH superam a influência da AOS no remodelamento ventricular esquerdo. As figuras 4 e 5 apresentam uma ampla dispersão das dimensões do átrio esquerdo e da aorta ascendente nos grupos sem e com AOS, o que demonstra que o tempo de evolução da apneia, bem como outros determinantes clínicos como idade e IMC podem contribuir para essas alterações estruturais cardíacas, apesar de não demonstrado nas análises multivariadas.

A AOS está intimamente ligada ao aparecimento de arritmias. A obesidade e a magnitude da dessaturação noturna de oxigênio associada com a AOS são fatores de risco independentes para o surgimento de FA em indivíduos com idade abaixo de 65 anos de idade. A FA é 5 vezes mais comum em pacientes com AOS do que em pacientes sem AOS. ${ }^{70}$ Nosso estudo também encontrou uma prevalência 5 vezes maior de FA em pacientes com MH e AOS em comparação com pacientes com $\mathrm{MH}$, mas sem AOS. Apesar da amostra relativamente pequena, o poder para detectar diferenças na prevalência de FA 
em pacientes com e sem AOS foi de $86 \%$. Os únicos fatores independentes associados com FA foram diâmetro do átrio esquerdo (em concordância com

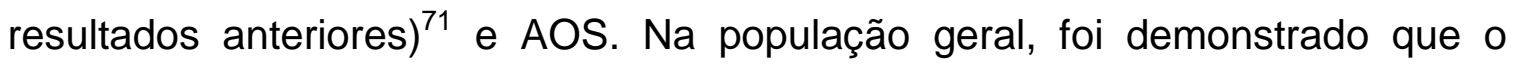
tratamento padrão da AOS com CPAP diminuiu a recorrência de FA, apontando para a importância desta relação. Este achado é particularmente importante, uma vez que existe uma clara relação entre FA e morte cardiovascular em pacientes com $\mathrm{MH}$. FA ocorre em $5 \%$ a $25 \%$ dos pacientes com $\mathrm{MH}$, aumenta com a idade e está ligada ao aumento atrial esquerdo..$^{12,13,16,30,72,73,74}$ Este estudo levanta a possibilidade de que o reconhecimento precoce e tratamento da AOS em pacientes com $\mathrm{MH}$ pode reduzir o risco cardiovascular. Um estudo recente identificou uma prevalência de FA maior nos indivíduos com $\mathrm{MH}$ e alteração na oximetria noturna sugestiva de AOS comparados aos indivíduos sem dessaturações noturnas ( $40 \%$ vs. $11 \%, p<0,01$; respectivamente) ${ }^{75}$ Além disso, os indivíduos com alteração de oximetria noturna apresentavam maior volume atrial esquerdo quando comparados aos indivíduos com $\mathrm{MH}$ e sem dessaturações noturnas (58 \pm 19 vs. $42 \pm 12 \mathrm{ml} / \mathrm{m}^{2}$, respectivamente). Esses achados são muito semelhantes aos resultados do nosso estudo e demonstram a consistência dos nossos dados.

Uma limitação do nosso estudo é o uso de um dispositivo de 4 canais que não analisa o sono. Assim, nossas medidas de IAH foram baseadas no tempo de gravação total e não no tempo total de sono, que pode ser reduzido em 
pacientes com insuficiência cardíaca. Por outro lado, este dispositivo foi recentemente validado contra polissonografia completa e, quando realizado em

casa, o estudo do sono pode melhor reproduzir o padrão de sono habitual. Além disso, a utilização de um exame de mais fácil execução em populações de alta prevalência aumenta a utilização dos nossos resultados na prática clínica. Outra limitação é que as hipopneias não podem ser distinguidas entre central e obstrutivas por causa do uso de uma cinta que utiliza um cristal piezoelétrico para medir excursões torácicas. Por outro lado, as apneias obstrutivas eram muito mais comuns do que as apneias centrais em todos os pacientes (tabela 4) e provavelmente também as hipopneias. Quanto ao ecocardiograma, um em cada quatro pacientes não teve a função diastólica avaliada por limitações técnicas e isso pode ter contribuído para o achado de que a função diastólica era similar entre o grupo sem e com AOS. A função diastólica alterada pode ser um dos mecanismos envolvidos no aumento atrial esquerdo nos pacientes com AOS. Devido à natureza transversal do presente estudo, não é possível concluir que a AOS está associada a futuras complicações cardiovasculares em pacientes com MH. Além disso, os pacientes com e sem AOS foram diferentes em relação a vários aspectos (Tabela 2) e não é possível assegurar que todas as variáveis de confusão foram totalmente ajustadas na análise multivariada. No entanto, nosso estudo está em concordância com os achados anteriores em outras populações ${ }^{57,76,77}$ e a associação independente entre a AOS e a FA aponta para a relevância dos nossos achados em pacientes com $\mathrm{MH}$. 
7 CONCLUSÃO 
1 AOS é altamente prevalente nos pacientes com $\mathrm{MH}$, apresentando uma prevalência de $40 \%$.

2 A AOS está associada com o aumento atrial esquerdo, um fator de risco para desenvolvimento de fibrilação atrial e óbito cardiovascular nessa população, e com o aumento da dimensão da aorta ascendente. No entanto, a AOS não apresentou associação com medidas de remodelamento ventricular.

3 A AOS é independentemente associada à $\mathrm{FA}$, um fator de risco para óbito cardiovascular nesta população.

Estes resultados sugerem que AOS deve ser investigada em pacientes com $\mathrm{MH}$, principalmente naqueles pacientes com maior idade, mais obesos e naqueles portadores de fibrilação atrial. Mais estudos são necessários para avaliar a segurança e o efeito do tratamento da AOS no remodelamento cardíaco e no surgimento de arritmias cardíacas nestes pacientes. 


\section{ANEXOS}




\begin{abstract}
ANEXO A
HOSPITAL DAS CLÍNICAS DA FACULDADE DE MEDICINA DA UNIVERSIDADE DE SÃO PAULO-HCFMUSP
\end{abstract}

TERMO DE CONSENTIMENTO LIVRE E ESCLARECIDO

DADOS DE IDENTIFICAÇÃO DO SUJEITO DA PESQUISA OU RESPONSÁVEL LEGAL

1. NOME:

DOCUMENTO DE IDENTIDADE № : SEXO:.$M \square \quad F$

DATA NASCIMENTO:

ENDEREÇO

№

APTO:

BAIRRO: CIDADE

CEP: ..TELEFONE: DDD (.... )

2.RESPONSÁVEL LEGAL

NATUREZA (grau de parentesco, tutor, curador etc.)

DOCUMENTO DE IDENTIDADE : SEXO: $M \square \quad F$ DATA NASCIMENTO.: ......./............. ENDEREÇO: № APTO:

BAIRRO: CIDADE:

CEP: TELEFONE: DDD ( 


\section{DADOS SOBRE A PESQUISA}

1. TÍTULO DO PROTOCOLO DE PESQUISA: Distúrbios do Sono na Miocardiopatia Hipertrófica: Prevalência, importância cardiovascular e possíveis implicações terapêuticas.

PESQUISADOR : Rodrigo Pinto Pedrosa

CARGO/FUNÇÃO: médico pesquisador. INSCRIÇÃO CONSELHO REGIONAL №114737

UNIDADE DO HCFMUSP: Laboratório do Sono do InCor da Disciplina de Pneumologia.

3. AVALIAÇÃO DO RISCO DA PESQUISA:

RISCO MÍNIMO X

RISCO BAIXO

RISCO MÉDIO

RISCO MAIOR

\section{HOSPITAL DAS CLÍNICAS DA FACULDADE DE MEDICINA DA UNIVERSIDADE DE SÃO PAULO-HCFMUSP}

O presente estudo que o $\mathrm{Sr}$ (a) está participando pretende identificar a presença da apneia do sono, que é uma doença que está associada a uma série de doenças do coração, como pressão alta, arritmias e derrame cerebral. Pouco se sabe da relação entre a apneia do sono e a Miocardiopatia Hipertrófica $(\mathrm{MH})$, que é a doença que o $\mathrm{Sr}$ (a) possui e que trata aqui no InCor. Queremos identificar a apneia por um exame do sono com um monitor portátil, parecido com o Holter, que os $\mathrm{Sr}$ (a) provavelmente já fez. Esse exame é indolor, simples e é feito em sua casa. O Sr (a) só terá que devolver o aparelho no outro dia do exame no Laboratório do Sono do InCor, 
no $7^{0}$ andar do Bloco I. Caso o Sr(a) seja portador da apneia, iremos ajudá-lo no seu tratamento, indicando a forma correta de tratá-lo(la). Os participantes que tiverem a apneia do sono e que apresentarem gradiente de pressão dentro do coração em decorrência da hipertrofia (uma condição comum e que causa falta de ar e pode causar arritmias) serão convidados a vir em um outro dia ao InCor para realização de um outro ecocardiograma usando o aparelho do CPAP, que é uma máquina que oferece um fluxo de ar para o nariz e que elimina a apneia, para avaliação do funcionamento do seu coração com esse aparelho, já que não se sabe muito sobre esse funcionamento em pacientes com $\mathrm{MH}$. O uso dessa máquina é bem tolerado e não gera incômodo na maioria dos pacientes que o utilizam. O Sr(a) não é obrigado a participar desse estudo e realizar esse exame do sono caso não seja de sua vontade, estando completamente livre a recusar e continuar seu tratamento no nosso ambulatório. Os dados da pesquisa serão analisados em conjunto, sendo totalmente confidencial os resultados dos seus exames. Todos os exames não terão nenhum custo para o senhor e ao final do estudo, o Sr(a) terá acesso aos resultados finais da nossa pesquisa e, se for diagnosticada a apneia do sono, será orientado quanto ao tratamento da mesma.

O principal investigador é o Dr. Rodrigo Pinto Pedrosa, que pode ser encontrado no Laboratório do Sono do Instituto do Coração, 7ํ, Bloco 1., Av. Dr. Enéias de Carvalho Aguiar, 44. Telefone: 11-30695486. Se você tiver alguma consideração ou dúvida sobre a ética da pesquisa, entre em contato com 0 Comitê de Ética em Pesquisa (CEP) - Rua Ovídio Pires de Campos, 225 - 5ํㅜㅇ andar - tel: 3069-6442 ramais 16, 17, 18 ou 20, FAX: 3069-6442 ramal 26 - E-mail: cappesq@hcnet.usp.br

É garantida a liberdade da retirada de consentimento a qualquer momento e deixar de participar do estudo, sem qualquer prejuízo à continuidade de seu tratamento na Instituição;

Acredito ter sido suficientemente informado a respeito das informações que li ou que foram lidas para mim, descrevendo o estudo "Distúrbios do 


\title{
Sono na Miocardiopatia Hipertrófica: Prevalência, importância cardiovascular e possíveis implicações terapêuticas."
}

\section{HOSPITAL DAS CLÍNICAS DA FACULDADE DE MEDICINA DA UNIVERSIDADE DE SÃO PAULO-HCFMUSP}

\begin{abstract}
Eu discuti com o Dr. Rodrigo Pinto Pedrosa sobre a minha decisão em participar nesse estudo. Ficaram claros para mim quais são os propósitos do estudo, os procedimentos a serem realizados, seus desconfortos e riscos, as garantias de confidencialidade e de esclarecimentos permanentes. Ficou claro também que minha participação é isenta de despesas e que tenho garantia do acesso a tratamento hospitalar quando necessário. Concordo voluntariamente em participar deste estudo e poderei retirar o meu consentimento a qualquer momento, antes ou durante o mesmo, sem penalidades ou prejuízo ou perda de qualquer benefício que eu possa ter adquirido, ou no meu atendimento neste Serviço.
\end{abstract}

Assinatura do paciente

Data 11

Assinatura da testemunha

Data $\frac{1}{1}$

analfabetos, semi-analfabetos ou portadores de deficiência auditiva ou visual.

(Somente para o responsável do projeto)

Declaro que obtive de forma apropriada e voluntária o Consentimento Livre e Esclarecido deste paciente ou representante legal para a participação neste estudo. 


\section{ANEXO B}

\section{Escala de sonolência de Epworth}

\begin{tabular}{|l|c|c|c|c|}
\hline SITUAÇÃO & \multicolumn{3}{|l|}{} \\
\hline Sentado e lendo. & 0 & 1 & 2 & 3 \\
\hline Assistindo TV. & 0 & 1 & 2 & 3 \\
\hline Sentado em um lugar público (cinema, igreja, sala de espera). & 0 & 1 & 2 & 3 \\
\hline $\begin{array}{l}\text { Como passageiro de trem, carro ou ônibus, andando uma } \\
\text { hora sem parar. }\end{array}$ & 0 & 1 & 2 & 3 \\
\hline Deitando-se para descansar à tarde, quando as & & & & \\
circunstâncias permitem. & & & & \\
\hline Sentado e conversando com alguém. & 0 & 1 & 2 & 3 \\
\hline Sentado calmamente após o almoço (sem álcool). & & & & \\
\hline $\begin{array}{l}\text { Dirigindo um carro, enquanto pára por alguns minutos ao } \\
\text { pegar um trânsito intenso. }\end{array}$ & 0 & 1 & 2 & 3 \\
\hline
\end{tabular}

Legendas: 0 - nenhuma chance de cochilar; 1 - pequena chance de cochilar; 2 moderada chance de cochilar; 3 - alta chance de cochilar.

Valores maiores que 10 pontos identificam indivíduos com significativa sonolência diurna. 


\section{ANEXO C}

Artigo publicado na Revista CHEST referente à presente TESE. 
9 REFERÊNCIAS 
1 Teare D. Asymmetrical hypertrophy of the heart in young adults. $\mathrm{Br}$ Heart J. 1958;20(1):1-8.

2 Braunwald E, Lambrew CT, Rockoff SD, Ross J Jr, Morrow AG. Idiopathic Hypertrophic Subaortic Stenosis. A description of the disease based upon an analysis of 64 patients. Circulation. 1964;30(Suppl 4):3-119.

3 Maron BJ. Hypertrophic cardiomyopathy. Lancet. 1997;350 (9071):127-33.

4 Miller MA, Gomes JA, Fuster V. Risk stratification of sudden cardiac death in hypertrophic cardiomyopathy. Nat Clin Pract Cardiovasc Med. 2007;4(12):667-6.

5 Zou Y, Song L, Wang Z, Ma A, Liu T, Gu H, Lu S, Wu P, Zhang dagger Y, Shen dagger L, Cai Y, Zhen double dagger Y, Liu Y, Hui R. Prevalence of idiopathic hypertrophic cardiomyopathy in China: a population-based echocardiographic analysis of 8080 adults. Am J Med. 2004;116(1):14-8.

6 Kubo T, Kitaoka H, Okawa M, Matsumura Y, Hitomi N, Yamasaki N, Furuno T, Takata J, Nishinaga M, Kimura A, Doi YL. Lifelong left ventricular remodeling of hypertrophic cardiomyopathy caused by a founder frameshift deletion mutation in the cardiac myosin-binding protein C gene among Japanese. J Am Coll Cardiol. 2005;46(9):1737-43. 
7 Maron MS, Olivotto I, Zenovich AG, Link MS, Pandian NG, Kuvin JT, Nistri S, Cecchi F, Udelson JE, Maron BJ. Hypertrophic cardiomyopathy is predominantly a disease of left ventricular outflow tract obstruction. Circulation. 2006;114(21):2232-9.

8 Wigle ED, Rakowski H, Kimball BP, Williams WG. Hypertrophic cardiomyopathy: Clinical spectrum and treatment. Circulation. 1995;92(7):1680-92.

9 Maron BJ, Gardin JM, Flack JM, Gidding SS, Kurosaki TT, Bild DE. Prevalence of hypertrophic cardiomyopathy in a general population of young adults. Echocardiographic analysis of 4111 subjects in the CARDIA Study. Coronary Artery Risk Development in (Young) Adults. Circulation. 1995;92(4)785-9.

10 Arteaga E, lanni BM, Fernandes F, Mady C. Benign outcome in a longterm follow-up of patients with hypertrophic cardiomyopathy in Brazil. Am Heart J. 2005;149(6):1099-105.

11 Shah PM, Adelman AG, Wigle ED, Gobel FL, Burchell HB, Hardarson T, Curiel R, De La Calzada C, Oakley CM, Goodwin JF. The natural (and unnatural) history of hypertrophic obstructive cardiomyopathy. Circ Res. 1974;35(2) (suppl II):179-95. 
12 Cecchi F, Olivotto I, Montereggi A, Santoro G, Dolara A, Maron BJ. Hypertrophic cardiomyopathy in Tuscany: clinical course and outcome in an unselected regional population. J Am Coll Cardiol. 1995;26(6):1529-36.

13 Maron BJ, McKenna WJ, Danielson GK, Kappenberger LJ, Kuhn HJ, Seidman CE, Shah PM, Spencer WH 3rd, Spirito P, Ten Cate FJ, Wigle ED. American College of Cardiology; Committee for Practice Guidelines. European Society of Cardiology. J Am Coll Cardiol. 2003;42(9):1687-713.

14 Maron BJ, Olivotto I, Bellone P, Conte MR, Cecchi F, Flygenring BP, Casey SA, Gohman TE, Bongioanni S, Spirito P. Clinical profile of stroke in 900 patients with hypertrophic cardiomyopathy. J Am Coll Cardiol. 2002;39(2):301-7.

15 Cha YM, Gersh BJ, Maron BJ, Boriani G, Spirito P, Hodge DO, Weivoda PL, Trusty JM, Friedman PA, Hammill SC, Rea RF, Shen WK. Electrophysiologic manifestations of ventricular tachyarrhythmias provoking appropriate defibrillator interventions in high-risk patients with hypertrophic cardiomyopathy. J Cardiovasc Electrophysiol. 2007;18(5):483-7. 
16 Olivotto I, Cecchi F, Casey SA, Dolara A, Traverse JH, Maron BJ. Impact of atrial fibrillation on the clinical course of hypertrophic cardiomyopathy. Circulation. 2001;104(21):2517-24.

17 Nistri S, Olivotto I, Betocchi S, Losi MA, Valsecchi G, Pinamonti B, Conte MR, Casazza F, Galderisi M, Maron BJ, Cecchi F. Prognostic significance of left atrial size in patients with hypertrophic cardiomyopathy (from the Italian Registry for Hypertrophic Cardiomyopathy). Am J Cardiol. 2006;98(7):960-5.

18 Yang H, Woo A, Monakier D, Jamorski M, Fedwick K, Wigle ED, Rakowski H. Enlarged left atrial volume in hypertrophic cardiomyopathy: a marker for disease severity. J Am Soc Echocardiogr. 2005;18(10):1074-82.

19 Seggewiss H, Faber L, Gleichmann U. Percutaneus transluminal septal ablation in hypertrophic obstructive cardiomyopathy. Thorac Cardiovasc Surg. 1999;47(2):94-100.

20 Daly MDB. Interactions between respiration and circulation. In: Cherniack NS, Widdicombe JG, editors. Handbook of Physiology. 3rd ed. Behesda: American Physiological Society, 1986:529-594.

21 Bradley TD, Floras JS. Sleep apnea and heart failure. Part I: Obstructive Sleep apnea. Circulation. 2003;107(12):1671-78. 
22 Sleep-related breathing disorders in adults: recommendations for syndrome definition and measurement techniques in clinical research. The Report of an American Academy of Sleep Medicine Task Force. Sleep. 1999;22(5):667-689.

23 Drager LF, Bortolotto LA, Figueiredo AC, Krieger EM, Lorenzi-Filho G. Effects of continuous positive airway pressure on early signs of atherosclerosis in obstructive sleep apnea. Am J Respir Crit Care Med. 2007;176(7):706-712.

24 Bradley TD, Floras JS. Obstructive sleep apnoea and its cardiovascular consequences. Lancet. 2009;373(9657):82-93.

25 Quan SF, Gersh BJ. Cardiovascular consequences of sleep disordered breathing: past, present and future. Circulation. 2004;109(8):951-7.

26 Collop NA, Anderson WM, Boehlecke B, Claman D, Goldberg R, Gottlieb DJ, Hudgel D, Sateia M, Schwab R. Portable Monitoring Task Force of the American Academy of sleep medicine, clinical guidelines for the use of unattended portable monitors in the diagnosis of obstructive sleep apnea in adult patients. Portable Monitoring task force of the American academy of sleep medicine. J Clin Sleep Med. 2007;3(7):737-47. 
27 Narkiewicz K, van de Borne P, Montano N, Dyken M, Phillips B, Somers V. Contribution of tonic chemoreflex activation to sympathetic activity and blood pressure in patients with obstructive sleep apnea. Circulation. 1998;97(10):943-945.

28 Young T, Peppard PE, Gottlieb DJ. Epidemiology of obstructive sleep apnea: a population health perspective. Am J Respir Crit Care Med. 2002;165(9):1217-39.

29 Gami AS, Pressman G, Caples SM, Kanagala R, Gard JJ, Davison DE, Malouf JF, Ammash NM, Friedman PA, Somers VK. Association of atrial fibrillation and obstructive sleep apnea. Circulation. 2004;110(4):364-7.

30 Drager LF, Bortolotto LA, Figueiredo AC, Silva BC, Krieger EM, LorenziFilho G. Obstructive sleep apnea, hypertension, and their interaction on arterial stiffness and heart remodeling. Chest. 2007;131:1379-86.

31 Drager LF, Bortolotto LA, Pedrosa RP, Krieger EM, Lorenzi-Filho G. Left atrial diameter is independently associated with arterial stiffness in patients with obstructive sleep apnea: potential implications for atrial fibrillation, Int J Cardiol 2009. [Epub ahead of print]

32 Parkash R, Green MS, Kerr CR, Connolly SJ, Klein GJ, Sheldon R, Talajic M, Dorian P, Humphries KH; Canadian Registry of Atrial Fibrillation. The association of left atrial size and occurrence of atrial fibrillation: a 
prospective cohort study from the Canadian Registry of Atrial Fibrillation. Am Heart J. 2004;148(4):649-54.

33 Logan AG, Perlikowski SM, Mente A, Tisler A, Tkacova R, Niroumand M, Leung RS, Bradley TD. High prevalence of unrecognized sleep apnoea in drug-resistant hypertension. J Hypertens. 2001;19(12):2271-7.

34 Bhama JK, Spagnolo S, Alexander EP, Greenberg M, Trachiotis GD. Coronary revascularization in patients with obstructive sleep apnea syndrome. Heart Surg Forum. 2006;9(6):E813-7.

35 Basner RC. Continuous Positive Airway Pressure for Obstructive Sleep Apnea. N Engl J Med. 2007;356(17):1751-1758.

36 Somers VK, Dyken ME, Clary MP, Abboud FM. Sympathetic neural mechanisms in obstructive sleep apnea. J Clin Invest. 1995;96(4):18971904.

37 Naughton MT, Rahman A, Hara K, Floras JS, Bradley TD. Effect of continuous positive airway pressure on intrathoracic and left ventricular transmural pressures in patients with congestive heart failure. Circulation. 1995;91(6):1725-1731.

38 Johnson CB, Beanlands RS, Yoshinaga K, Haddad H, Leech J, de Kemp R, Burwash IG. Acute and chronic effects of continuous positive airway pressure therapy on left ventricular systolic and diastolic function in 
patients with obstructive sleep apnea and congestive heart failure. Can $J$ Cardiol. 2008;24(9):697-704.

39 Banno K, Shiomi T, Sasanabe R, Otake K, Hasegawa R, Maekawa M, Ito T. Sleep-Disordered Breathing in Patients With Idiopathic Cardiomyopathy. Circ J. 2004;68(4):338-342.

40 Sengupta PP, Sorajja D, Eleid MF, Somers VK, Ommen SR, Parish JM, Khandheria B, Tajik AJ. Hypertrophic obstructive cardiomyopathy and sleep-disordered breathing: an unfavorable combination. Nat Clin Pract Cardiovasc Med. 2009;6(1):14-5.

41 Sin DD, Fitzgerald F, Parker JD, Newton G, Floras JS, Bradley TD. Risk factors for central and obstructive sleep apnea in 450 men and women with congestive heart failure. Am $J$ Respir Crit Care Med. 1999;160(4):1101-1106.

42 Lanfranchi PA, Braghiroli A, Bosimini E, Mazzuero G, Colombo R, Donner CF, Giannuzzi P. Prognostic value of nocturnal Cheyne-Stokes respiration in chronic heart failure. Circulation. 1999;99(11):1435-1440.

43 Hanly PJ, Milar TW, Steljes DG, Beart R, Frais MA, Kryger MH. Respiration and abnormal sleep in patients with congestive heart failure. Chest. 1989; 96(3): 480-488. 
44 Lorenzi-Filho G, Dajani HR, Leung RST, Floras JS, Bradley TD. Entrainment of blood pressure and heart rate oscillations by periodic breathing. Am J Respir Crit Care Med. 1999;159(4):1114-1154.

45 Trinder J, Merson R, Rosemberg J, Fitzgerald F, Kleiman J, Bradley TD. Pathophysiological interactions of ventilation, arousals, and blood pressure oscillations during cheyne-stokes respiration in patients with heart failure. Am J Respir Crit Care Med. 2000;162(3 Pt 1):808-13.

46 Javaheri S, Corbett WS. Association of low $\mathrm{PaCO} 2$ with central sleep apnea and ventricular arrhythmias in ambulatory patients with stable heart failure. Ann Intern Med. 1998;128(3):204-7.

47 Hanly PJ, Zuberi-Khokhar N. Daytime sleepiness in patients with congestive heart failure and Cheyne-Stokes respiration. Chest. 1995;107(4):952-958.

48 Dowdell WT, Javaheri S, McGinnis W. Cheyne-Stokes respiration presenting as sleep apnea syndrome. Clinical and polysomnographic features. Am Rev Respir Dis. 1990; 141(4 Pt 1): 871-879.

49 Hanly PJ, Zuberi-Kokhar NS. Increased mortality associated with CheyneStokes respiration in patients with congestive heart failure. Am J Respir Crit Care Med. 1996;153(1):272-276. 
50 Young T, Palta M, Dempsey J, Skatrud J, Weber S, Badr S. The occurrence of sleep-disordered breathing among middle-aged adults. $N$ Engl J Med. 1993;29;328(17):1230-5.

51 Johns MW. A new method for measuring daytime sleepiness: the Epworth sleepiness scale. Sleep. 1991;14(6):540-545.

52 Santos-Silva R, Sartori DE, Truksinas V, Truksinas E, Alonso FF, Tufik S, Bittencourt LR. Validation of a portable monitoring system for the diagnosis of obstructive sleep apnea syndrome. Sleep. 2009;32(5):62936.

53 American Academy of Sleep Medicine. The AASM manual for the scoring of sleep and associated events. Westchester, IL: American Academy of Sleep Medicine, 2007.

54 Lang RM, Bierig M, Devereux RB, Flachskampf FA, Foster E, Pellikka PA, Picard MH, Roman MJ, Seward J, Shanewise J, Solomon S, Spencer KT, St John Sutton M, Stewart W; American Society of Echocardiography's Nomenclature and Standards Committee; Task Force on Chamber Quantification; American College of Cardiology Echocardiography Committee; American Heart Association; European Association of Echocardiography, European Society of Cardiology. Recommendations for chamber quantification. Eur J Echocardiogr. 2006;7(2):79-108. 
55 Oh JK, Appleton CP, Hatle LK, Nishimura RA, Seward JB, Tajik AJ . The noninvasive assessment of left ventricular diastolic function with twodimensional and Doppler echocardiography. J Am Soc Echocardiogr. 1997;10( 3 ):246 - 270.

56 Eleid MF, Konecny T, Orban M, Sengupta PP, Somers VK, Parish JM, Mookadam F, Brady PA, Sullivan BL, Khandheria BK, Ommen SR, Tajik AJ. High prevalence of abnormal nocturnal oximetry in patients with hypertrophic cardiomyopathy. J Am Coll Cardiol. 2009;54(19):1805-9.

57 Peppard PE, Young T, Palta M, Skatrud J. Prospective study of the association between sleep-disordered breathing and hypertension. N Engl J Med. 2000;342(19):1378-84.

58 Arzt M, Young T, Finn L, Skatrud JB, Ryan CM, Newton GE, Mak S, Parker JD, Floras JS, Bradley TD. Sleepiness and sleep in patients with both systolic heart failure and obstructive sleep apnea. Arch Intern Med. 2006;166(16):1716-22.

59 Elias RM, Castro MC, de Queiroz EL, Abensur H, Romão JE Jr, LorenziFilho G. Obstructive sleep apnea in patients on conventional and short daily hemodialysis. Am J Nephrol. 2009;29(6):493-500. 
60 Chiu KL, Ryan CM, Shiota S, Ruttanaumpawan P, Arzt M, Haight JS, Chan CT, Floras JS, Bradley TD. Fluid shift by lower body positive pressure increases pharyngeal resistance in healthy subjects. Am $J$ Respir Crit Care Med. 2006;174(12):1378-83.

61 Shiota S, Ryan CM, Chiu KL, Ruttanaumpawan P, Haight J, Arzt M, Floras JS, Chan C, Bradley TD. Alterations in upper airway cross-sectional area in response to lower body positive pressure in healthy subjects. Thorax. 2007;62(10):868-72 .

62 Drager LF, Queiroz EL, Lopes HF, Genta PR, Krieger EM, Lorenzi-Filho G. Obstructive Sleep Apnea is highly prevalent and correlates with poor glycemic control in consecutive patients with Metabolic Syndrome. $J$ Cardiometab Syndr. 2009;4(2):89-95.

63 Drager LF, Bortolotto LA, Maki-Nunes C, Trombetta IC, Alves MJ, Fraga RF, Negrão CE, Krieger EM, Lorenzi-Filho G. The incremental role of obstructive sleep apnoea on markers of atherosclerosis in patients with metabolic syndrome. Atherosclerosis. 2010;208(2):490-5.

64 Garrigue S, Pépin JL, Defaye P, Murgatroyd F, Poezevara Y, Clémenty J, Lévy P. High prevalence of sleep apnea syndrome in patients with longterm pacing: the European Multicenter Polysomnographic Study. Circulation. 2007;115(13):1703-9. 
65 Drager LF, Genta PR, Pedrosa RP, Nerbass FB, Gonzaga CC, Krieger EM, Lorenzi-Filho G. Characteristics and predictors of obstructive sleep apnea in patients with systemic hypertension. $A m J$ Cardiol. 2010;105(8):1135-9.

66 Drager LF, Lopes HF, Maki-Nunes C, Trombetta IC, Toschi-Dias E, Alves MJ, Fraga RF, Jun JC, Negrão CE, Krieger EM, Polotsky VY, LorenziFilho G. The impact of obstructive sleep apnea on metabolic and inflammatory markers in consecutive patients with metabolic syndrome. PLoS One. 2010;5(8).pii: e12065.

67 Pedrosa RP, Drager LF, Antunes MO, Arteaga E, Lorenzi-Filho L. Cheyne-Stokes Respiration associated with hypertrophic cardiomyopathy and normal left ventricular ejection fraction. Clinics. 2010. (in press)

68 Usui K, Parker JD, Newton GE, Floras JS, Ryan CM, Bradley TD. Left ventricular structural adaptations to obstructive sleep apnea in dilated cardiomyopathy. Am J Respir Crit Care Med 2006;173:1170-5.

69 Serizawa N, Yumino D, Takagi A, Gomita K, Kajimoto K, Tsurumi Y, Hagiwara N. Obstructive sleep apnea is associated with greater thoracic aortic size. J Am Coll Cardiol. 2008;52(10):885-6.

70 Mehra R, Benjamin EJ, Shahar E, Gottlieb DJ, Nawabit R, Kirchner HL, Sahadevan J, Redline S. Association of nocturnal arrhythmias with sleep- 
disordered breathing: The Sleep Heart Health Study. Am J Respir Crit Care Med. 2006;173(8):910-16.

71 Cui $Q$, Zhang $W$, Wang $H$, Sun $X$, Yang $H$, Meng $X$, Zhang $Y$, Wang $H$. Left and right atrial size and the occurrence predictors in patients with paroxysmal atrial fibrillation. Int J Cardiol. 2008;130(1):69-71.

72 Maron BJ, Casey SA, Poliac LC, Gohman TE, Almquist AK, Aeppli DM. Clinical course of hypertrophic cardiomyopathy in a regional United States cohort. JAMA. 1999;281(7):650-5.

73 Robinson K, Frenneaux MP, Stockins B, Karatasakis G, Poloniecki JD, McKenna WJ. Atrial fibrillation in hypertrophic cardiomyopathy: a longitudinal study. J Am Coll Cardiol. 1990;15(6):1279-85.

74 Hematpour K, Steinberg JS. Treatment of atrial fibrillation in hypertrophic cardiomyopathy. Anadolu Kardiyol Derg. 2006;6 (Suppl 2):44-8.

75 Konecny T, Brady PA, Orban M, Lin G, Pressman GS, Lehar F, Tomas K, Gersh BJ, Tajik AJ, Ommen SR, Somers VK. Interactions between sleep disordered breathing and atrial fibrillation in patients with hypertrophic cardiomyopathy. Am J Cardiol. 2010;105(11):1597-602.

76 Marin JM, Carrizo SJ, Vicente E, Agusti AG. Long-term cardiovascular outcomes in men with obstructive sleep apnea- hypopnea with or without 
treatment with continuous positive airway pressure: an observational study. Lancet. 2005;365(9464):1046-53.

77 Young T, Finn L, Peppard PE, Szklo-Coxe M, Austin D, Nieto FJ, Stubbs R, Hla KM. Sleep disordered breathing and mortality: eighteen-year followup of the Wisconsin sleep cohort. Sleep. 2008;31(8):1071-8. 